Rev. Mat. Iberoamericana 26 (2010), no. 2, 651-692

\title{
Bernstein-Heinz-Chern results in calibrated manifolds
}

\author{
Guanghan Li and Isabel M. C. Salavessa
}

\begin{abstract}
Given a calibrated Riemannian manifold $\bar{M}$ with parallel calibration $\Omega$ of rank $m$ and $M$ an orientable m-submanifold with parallel mean curvature $H$, we prove that if $\cos \theta$ is bounded away from zero, where $\theta$ is the $\Omega$-angle of $M$, and if $M$ has zero Cheeger constant, then $M$ is minimal. In the particular case $M$ is complete with $\operatorname{Ricci}^{M} \geq 0$ we may replace the boundedness condition on $\cos \theta$ by $\cos \theta \geq C r^{-\beta}$, when $r \rightarrow+\infty$, where $0<\beta<1$ and $C>0$ are constants and $r$ is the distance function to a point in $M$. Our proof is surprisingly simple and extends to a very large class of submanifolds in calibrated manifolds, in a unified way, the problem started by Heinz and Chern of estimating the mean curvature of graphic hypersurfaces in Euclidean spaces. It is based on an estimation of $\|H\|$ in terms of $\cos \theta$ and an isoperimetric inequality. In a similar way, we also give some conditions to conclude $M$ is totally geodesic. We study some particular cases.
\end{abstract}

\section{Introduction}

E. Heinz [18] in 1955 introduced the problem of estimating the mean curvature of a surface of $\mathbb{R}^{3}$ described by a graph of a function $f: \mathbb{R}^{2} \rightarrow \mathbb{R}$. He proved that if $f$ is defined on the disc $x^{2}+y^{2}<r^{2}$ and the mean curvature satisfies $\|H\| \geq c>0$, where $c$ is a constant, then $r \leq \frac{1}{c}$. Thus, if $f$ is defined in all $\mathbb{R}^{2}$ and $\|H\|$ is constant, then $H=0$. Later, this result was extended for the case of a map $f: \mathbb{R}^{m} \rightarrow \mathbb{R}$ by Chern [9] and independently, by Flanders [15]. This problem was generalized by the second author in her Ph.D thesis ([29], [30]) in 1987, for submanifolds of a Riemannian product

2000 Mathematics Subject Classification: 53C42, 53C38, 53C40, 58E35.

Keywords: Calibrated geometry, parallel mean curvature, Heinz-inequality, Bernstein. 
$\bar{M}=M \times N$ of Riemannian manifolds $\left(M, g_{1}\right)$ and $(N, h)$, that can be described as a graph $\Gamma_{f}:=\{(p, f(p)): p \in M\}$ of a smooth map $f: M \rightarrow N$, that we recall as follows. On any oriented Riemannian manifold $(M, g)$ it is defined an isoperimetric constant, the Cheeger constant

$$
\mathfrak{h}(M, g)=\inf _{D} \frac{A(\partial D, g)}{V(D, g)}
$$

where $D$ ranges over all open submanifolds of $M$ with compact closure in $M$ and smooth boundary (see e.g. [7] and Section 4), and $A(\partial D, g)$ and $V(D, g)$ are respectively the area of $\partial D$ and the volume of $D$, with respect to the metric $g$. We call such $D$ by compact domain. The Cheeger constant is zero, if, for example, $M$ is a closed manifold (we abusively take the same definition for the closed case), or if $M$ is a simple Riemannian manifold, that is, there exists a diffeomorphism $\phi:(M, g) \rightarrow\left(\mathbb{R}^{m},<,>\right)$ onto $\mathbb{R}^{m}$ such that $\lambda^{2} g \leq \phi^{*}<,>\leq \mu^{2} g$ for some positive constants $\lambda, \mu$. Another large class of Riemannian manifolds with zero Cheeger constant are the complete Riemannian manifolds with non-negative Ricci tensor (see Section 4). Hence, zero Cheeger constant is a quite interesting condition.

Theorem 1.1. ([29,30]) If $f:\left(M, g_{1}\right) \rightarrow(N, h)$ is a smooth map whose graph $\Gamma_{f}$ has parallel mean curvature $H$, then for each compact domain $D \subset M$ we have the isoperimetric inequality

$$
\|H\| \leq \frac{1}{m} \frac{A\left(\partial D, g_{1}\right)}{V\left(D, g_{1}\right)} .
$$

Thus $\|H\| \leq \frac{1}{m} \mathfrak{h}\left(M, g_{1}\right)$. In particular if $\left(M, g_{1}\right)$ has zero Cheeger constant then $\Gamma_{f}$ is a minimal submanifold of $M \times N$.

We may also handle this problem in the context of calibrated manifolds. A calibration on a Riemannian manifold $\bar{M}$ of dimension $m+n$ is a closed $m$-form $\Omega$ with comass one, that is, for each $p \in \bar{M}$ and any orthonormal system $X_{i} \in T_{p} \bar{M},\left|\Omega\left(X_{1}, \ldots, X_{m}\right)\right| \leq 1$ holds, and equality is achieved at some system (see [17]). If $F: M \rightarrow \bar{M}$ is an oriented immersed submanifold of dimension $m$, it is defined the $\Omega$-angle of $M, \theta: M \rightarrow[0, \pi]$, given by

$$
\cos \theta=\Omega\left(X_{1}, \ldots, X_{m}\right)
$$

where $X_{i}$ is a direct orthonormal frame of $T_{p} M$. We give to $M$ the induced metric $g=F^{*} \bar{g}$. The submanifold is said to be $\Omega$-calibrated if $\cos \theta=1$. This is equivalent to $\Omega$ restricted to $M$ is the volume element of $M$. Calibrated submanifolds are minimal, for they minimize the volume of any domain $D$ among all variations $F_{t}: D \rightarrow \bar{M}, t \in[0,1]$, of $F_{0}=F$ that fixes the 
boundary $\partial D$. Let $d V_{t}$ be the volume element of $\left(D, g_{t}=F_{t}^{*} \bar{g}\right)$. Assuming $F_{0}$ is calibrated, integration over $D$ of

$$
\cos \theta_{1} d V_{1}-d V_{0}=F_{1}^{*} \Omega-F_{0}^{*} \Omega=d \tau
$$

where $\tau=\int_{0}^{1} F_{t}^{*}\left(\Omega\left(\frac{\partial F}{\partial t}, \cdot\right)\right) d t$ is a $(m-1)$-form that satisfies $\tau_{\left.\right|_{\partial D}}=0$, gives

$$
V_{1}(D) \geq \int_{D} \cos \theta_{1} d V_{1}=\int_{D} d V_{0}=V_{0}(D) .
$$

This inequality shows $F_{0}$ is minimal. Furthermore, if $F_{1}$ also minimizes the volume on the homotopy class of a calibrated submanifold, then $F_{1}$ is a calibrated submanifold as well. On the other hand, a stable minimal submanifold $F$ may not be $\Omega$-calibrated. This is the case $\bar{M}$ has two different $m$-calibrations and $F$ is calibrated only for one of them. A pertinent question is to ask when is it true that stable minimal submanifolds are in fact calibrated for some calibration. This is true at least locally, for hypersurfaces in Euclidean spaces, or more generally for submanifolds under certain integrability conditions (see Subsection 5.2).

The simplest examples of Riemannian manifolds with a calibration are the Riemannian products $\bar{M}=\left(M \times N, g_{1} \times h\right)$, with the volume calibration

$$
\Omega\left(\left(X_{1}, Y_{1}\right), \ldots,\left(X_{m}, Y_{m}\right)\right)=\operatorname{Vol}_{\left(M, g_{1}\right)}\left(X_{1}, \ldots, X_{m}\right) .
$$

If $M$ is a graph submanifold $\Gamma_{f}: M \rightarrow M \times N$ then

$$
\cos \theta=\left(\operatorname{det}\left(g_{1}+f^{*} h\right)\right)^{-1 / 2}>0,
$$

where the determinant is with respect to the metric $g_{1}$. Reciprocally, a $m$ dimensional submanifold of $M \times N$ is locally a graph if $\cos \theta>0$. The graph is a calibrated submanifold if and only if $f$ is constant, that is, the graph is a slice. The condition $\cos \theta \geq \tau>0, \tau$ a constant, is equivalent to the boundedness of $\|d f\|^{2}$. The induced metric on the graph $M$ is the graph metric $g=g_{1}+f^{*} h$ on $M$ and so, under the above condition the metrics $g$ and $g_{1}$ are equivalent. In this case, $(M, g)$ has zero Cheeger constant if and only if $\left(M, g_{1}\right)$ has so.

In this paper we will obtain the result in Theorem 1.1 from a general result for any calibration $\Omega$, but with an extra condition on $\cos \theta$ at infinity. This means that this approach for graphs is not so good has the one in $[29,30]$, although they are very much related to each other. In both approaches we use a suitable vector field $Z_{1}$, naturally defined on all $M$ using the calibration, but in Theorem 1.1 we consider the divergence of $Z_{1}$ with respect to the metric $g_{1}$ of $M$, while in next theorem we consider the divergence with respect to the induced metric $g$ of $M$. On the other hand, we will 
provided a unified way to obtain a Heinz-Chern result for submanifolds with parallel mean curvature in a very large class of ambient spaces, the class of calibrated manifolds.

Examples of calibrated manifolds are the Kähler manifolds with the Kähler calibration, the Riemanniam manifolds with special holonomy, namely, the Calabi-Yau manifolds with the special Lagrangian calibration, the quaternionic-Kähler manifolds with the quaternionic calibration, the hyperKähler manifolds (with many calibrations), $G_{2}$ manifolds with the associative and co-associative calibration, and $\operatorname{Spin}(7)$ manifolds with the Cayley calibration (see [22]). These special spaces are Einstein manifolds, and except the quaternionic-Kähler case, they are all Ricci flat. If $n=1$, a parallel $\Omega$ defines a non-zero global parallel vector field $(* \Omega)^{\sharp}$ on $\bar{M}$ and so, if $\bar{M}$ is simply connected then it splits as a Riemannian product $\bar{M}=M \times N^{1}$, where $N^{1}$ is one dimensional, and $\Omega$ is the volume element of $M$. More generally, for $n=1$, a divergence free vector field $\bar{X}$ on $\bar{M}$, defines a closed $m$-form $\Omega=* \bar{X}^{b}$, where $*$ is the star operator on $\bar{M}$. This form is a calibration if $\|\bar{X}\|=1$. This is the case of a Riemannian manifold $\bar{M}$ with a codimension-one transversally oriented foliation by minimal hypersurfaces, for, in this case the unit normal $\bar{X}$ to the leaves defines a divergence free vector field of $\bar{M}$ that calibrates the leaves. For foliations of any codimension see Section 5 .

In what follows, $(\bar{M}, \bar{g}, \Omega)$ denotes a calibrated $(m+n)$-dimensional manifold with a calibration $\Omega$ of rank $m \geq 2$, and $F: M \rightarrow \bar{M}$ is an immersed oriented submanifold of dimension $m$, induced metric $g$, volume element $d V$, normal bundle $N M$, mean curvature $H$ and $\Omega$-angle $\theta$. We consider the following morphisms $\Phi: T M \rightarrow N M, \Psi: \wedge^{2} T M \rightarrow \wedge^{2} N M$, such that for $X, Y \in T_{p} M, U, V \in N M_{p}$,

$$
\begin{array}{ll}
\bar{g}(\Phi(X), U) & =\Omega(U, * X), \\
\langle\Psi(X \wedge Y), U \wedge W\rangle & =\Omega(U \wedge W, * X \wedge Y),
\end{array}
$$

where $*: T M \rightarrow \wedge^{m-1} T M$ and $*: \wedge^{2} T M \rightarrow \wedge^{m-2} T M$ are the star operators and $\langle$,$\rangle is the usual inner product in \wedge^{2} N M$. For $m=2$, set $\langle\Psi(d V), U \wedge W\rangle=\Omega(U \wedge W)$, where $d V$ is the volume element of $M$. Our main results are:

Theorem 1.2. (The integral $\Omega$-isoperimetric inequality). On a compact domain $D$ of $M$, with boundary $\partial D$ with volume element $d A$, the following inequality holds

$$
\left|\int_{D}\left(-m \cos \theta\|H\|^{2}+\left\langle\nabla^{\perp} H, \Phi\right\rangle\right) d V+\int_{D} \bar{\nabla}_{H} \Omega\right| \leq \int_{\partial D} \sin \theta\|H\| d A,
$$

where $\langle$,$\rangle is the Hilbert-Schmidt inner product in T M^{*} \otimes N M$. 
From now on we assume $\Omega$ is parallel. Theorem 1.2 leads to:

Theorem 1.3. If $F: M \rightarrow \bar{M}$ is immersed with parallel mean curvature and $\cos \theta>0$, on a compact domain $D$ of $M$, the following isoperimetric inequality holds:

$$
\|H\| \leq \frac{1}{m}\left(\frac{\sup _{\partial D} \sin \theta}{\inf _{D} \cos \theta}\right) \frac{A(\partial D, g)}{V(D, g)}
$$

In particular:

(1) If $\cos \theta \geq \tau>0$ where $\tau$ is a constant, then $\|H\| \leq \frac{1}{m} \frac{\sqrt{1-\tau^{2}}}{\tau} \mathfrak{h}(M, g)$. In this case, if $M$ has zero Cheeger constant, then $M$ is a minimal submanifold.

(2) If $\cos \theta=1$ on $\partial D$ for some domain $D$ then $F$ is a minimal immersion.

Corollary 1.1. If $M$ is closed with parallel mean curvature and $\cos \theta>0$, then $M$ is minimal.

Corollary 1.2. If $M$ is closed, $1>|\cos \theta|$ constant, and $\|\Phi(X)\| \leq \mu \sin \theta\|X\|$, where $0<\mu \leq 1$ is a constant, and $\|H\|$ not identically zero, then

$$
|\cot \theta| \leq \frac{\mu}{\sqrt{m}} \frac{\int_{M}\left\|\nabla^{\perp} H\right\| d V}{\int_{M}\|H\|^{2} d V} .
$$

Equality holds iff $\Phi$ is a homothetic morphism with coefficient of conformality $\mu^{2} \sin ^{2} \theta$ on the orthogonal complement of the distribution defined by the kernel of $\nabla^{\perp} H$, and $\nabla_{X}^{\perp} H=\Phi(\psi(X))$ where $\psi: T M \rightarrow T M$ is a linear morphism.

We will see that $\|\Phi(X)\| \leq \sin \theta\|X\|$ always hold. The conformality condition on $\Phi$ is not an uncommon condition. In a 8-dimensional quaternionic Kähler manifold, almost complex 4-submanifolds define a morphism $\Phi$ with coefficient of conformality $(1-\cos \theta)\left(\cos \theta-\frac{1}{3}\right)$. Four dimensional submanifolds with equal Kähler angles of a Kähler manifold of complex dimension 4, define $\Phi$ with coefficient of conformality $(1-\cos \theta) \cos \theta$ (see Section 5).

We can slightly improve Theorem 1.3 in case $\operatorname{Ricci}^{M} \geq 0$ and $M$ is complete. In this case, if we fix $p \in M$, there is a constant $C_{1}>0$, such that (see Section 4)

$$
\mathfrak{h}(M) \leq \mathfrak{h}\left(B_{r}(p)\right) \leq \frac{C_{1}}{r} \quad \text { for all } r \in(0,+\infty)
$$

Theorem 1.4. If $F: M \rightarrow \bar{M}$ is a complete immersed oriented m-dimensional submanifold with parallel mean curvature, and Ricci $^{M} \geq 0$ and the $\Omega$-angle satisfies $\cos \theta \geq C r^{-\beta}>0$ when $r \rightarrow+\infty$, where $0 \leq \beta<1$ and $C>0$ are constants, and $r$ is the distance function in $M$ to a point $p \in M$, then $F$ is a minimal submanifold. 
An application of Theorem 1.1 is the following:

Proposition 1.1. If $\left(M, g_{1}\right)$ is a complete Riemannian manifold with Ri$c c i^{\left(M, g_{1}\right)} \geq 0$, then any graphic submanifold with parallel mean curvature $F=\Gamma_{f}: M \rightarrow\left(M \times N, g_{1} \times h\right)$, where $f:\left(M, g_{1}\right) \rightarrow(N, h)$ is a smooth map, is a minimal submanifold.

It is fundamental some nonnegativeness on the curvature tensor of $M$ to obtain such Heinz-Chern results. If $\bar{M}=\mathbb{H}^{m} \times \mathbb{R}$ where $\mathbb{H}^{m}$ is is the $m$ hyperbolic space, there are examples of entire graphic hypersurfaces, and so complete, with non-zero constant mean curvature $c$ and with $\cos \theta$ bounded away from zero, as can be shown by the following proposition. Note that $\mathfrak{h}\left(\mathbb{H}^{m}\right)=m-1$. The function $r(x)=\ln ((1+|x|) /(1-|x|))$ is the distance function in $\mathbb{H}^{m}$ to 0 , for the Poincaré model, and $\nu=(-\nabla f, 1) / \sqrt{1+\|\nabla f\|^{2}}$ is a unit normal to $\Gamma_{f}$ :

Proposition 1.2. $[29,30,32]$ For each $|c| \leq m-1, f_{c}: \mathbb{H}^{m} \rightarrow \mathbb{R}$ defined by:

$$
f_{c}(x)=\int_{0}^{r(x)} \frac{\frac{c}{(\sinh r)^{m-1}} \int_{0}^{r}(\sinh t)^{m-1} d t}{\sqrt{1-\left(\frac{c}{(\sinh r)^{m-1}} \int_{0}^{r}(\sinh t)^{m-1} d t\right)^{2}}} d r
$$

is smooth on all $\mathbb{H}^{m}$, and for each $d \in \mathbb{R}, \Gamma_{f_{c}+d} \subset \mathbb{H}^{m} \times \mathbb{R}$ has constant mean curvature given by $\bar{g}(H, \nu)=\frac{c}{m}$, and $\cos \theta>\sqrt{(m-1-|c|) /(m-1)}$. Furthermore, $\left\{\Gamma_{\left(f_{c}\right)+d}(x): x \in \mathbb{H}^{m}, d \in \mathbb{R}\right\}$ and $\left\{\Gamma_{\left(f_{c}\right)+d}(x): x \in \mathbb{H}^{m}, c \in\right.$ $[1-m, m-1]\}$ define (partial) foliations of $\mathbb{H}^{m} \times \mathbb{R}$ by hypersurfaces with the same constant mean curvature $c$, and with constant mean curvature parameterized by the leaf, respectively.

A related classical problem is the Bernstein-type problem, that determines when a minimal submanifold must be totally geodesic. In 1927, Bernstein [6] proved that any minimal surface of $\mathbb{R}^{3}$ defined by the graph of an entire map $f: \mathbb{R}^{2} \rightarrow \mathbb{R}$ is a linear plane. This result was generalized to $\mathbb{R}^{m+1}$ for $m \leq 7$ by De Giorgi [11] $(m=3)$, Almgren [2] $(m=4)$, and Simons [36] $(m \leq 7)$, and to higher dimensions and codimensions under various growth conditions by many others, as for example Hildebrandt, Jost, and Widmann in [19], Ecker and Huisken [13], Wang [37], and more recently some attention is given to Bernstein theorems in curved Riemannian product or warped product spaces by Alías, Dajczer and Ripoll [3]. In higer dimension, and mainly in higer codimension, Bernstein-type results tend to be more difficult and complicated to formulate. Some Bernstein results have been obtained for stable minimal hypersurfaces by Do Carmo and Peng [12], Miranda [27], Fischer-Colbrie, Schoen, Simon and Yau [14, 35], and for leaves 
of transversely oriented codimension one foliations of Riemannian manifolds by Barbosa, Kenmotsu, Oshikiri, Bessa and Montenegro [5, 4], where ChernHeinz inequalities are derived, as well the stability of the leaves.

In this paper we obtain some Bernstein-type results using the same philosophy of the Chern-Heinz inequalities, applied to submanifolds immersed in calibrated manifolds, and under certain conditions, allowing us to obtain this type of results in any codimension. They are derived from the expression of $\Delta \cos \theta$. This Laplacian involves the covariant derivative of the mean curvature, a quadratic term on the second fundamental form $B$ and a curvature term of $\bar{M}$ that we have to analyse. We should have in mind that if $F$ is totally geodesic then $\theta$ is constant. Let $B_{\Phi}$ a 1 -form on $M$ and $\wedge^{2} B: \wedge^{2} T M \rightarrow \wedge^{2} N M$ given by

$$
\begin{aligned}
B_{\Phi}(X) & =\sum_{i} \bar{g}\left(B\left(X_{i}, X\right), \Phi\left(X_{i}\right)\right) \\
\wedge^{2} B(X \wedge Y) & =\sum_{i} B\left(X_{i}, X\right) \wedge B\left(X_{i}, Y\right),
\end{aligned}
$$

where $X_{i}$ is any orthonormal basis of $T_{p} M$. We consider the following quadratic forms defined for any $m$-calibration $\Omega$ and $F: M^{m} \rightarrow \bar{M}$ satisfying $\cos \theta>0$, and applied to tensors $B^{\prime} \in \bigodot^{2} T M^{*} \otimes N M$

$$
\begin{aligned}
& Q_{\Omega}\left(B^{\prime}\right)=\tilde{Q}_{\Omega}\left(B^{\prime}\right)+\frac{1}{\cos ^{2} \theta}\left\|B_{\Phi}^{\prime}\right\|^{2} \\
& \tilde{Q}_{\Omega}\left(B^{\prime}\right)=\left\|B^{\prime}\right\|^{2}-\frac{2}{\cos \theta}\left\langle\Psi, \wedge^{2} B^{\prime}\right\rangle .
\end{aligned}
$$

$Q_{\Omega}\left(\right.$ or $\left.\tilde{Q}_{\Omega}\right)$ is said to be $\delta$-positive at $B^{\prime}$ if $Q_{\Omega}\left(B^{\prime}\right) \geq \delta\left\|B^{\prime}\right\|^{2}$, where $\delta>0$. This is the case, with $\delta=1$, when $\left\langle\Psi, \wedge^{2} B^{\prime}\right\rangle \leq 0$, as it is the case $n=1$.

The quadratic form $Q_{\Omega}$ was defined in [37] for the case $\bar{M}=M^{m} \times N^{n}$ and $\Omega$ the volume element of $\left(M, g_{1}\right)$ and $F=\Gamma_{f}$ with $f:\left(M, g_{1}\right) \rightarrow(N, h)$ a smooth map. For $n \geq 2$, one has to require $\lambda_{i} \lambda_{j} \leq(1-\delta)$, for $i \neq j$ and some constant $0<\delta \leq 1$, where $\lambda_{1}^{2} \geq \ldots \geq \lambda_{m}^{2} \geq 0$ are the eigenvalues of $f^{*} h$, to have $Q_{\Omega} \delta$-positive at any $B^{\prime}$. This condition gives bounds to the components of the calibration $\Omega$ in a convenient basis $X_{i}$ of $T M$ and $U_{\alpha}$ of $N M$, namely on the components $\left\langle\Psi\left(X_{i} \wedge X_{j}\right), U_{\alpha} \wedge U_{\beta}\right\rangle=\cos \theta \delta_{i \alpha} \delta_{j \beta} \lambda_{i} \lambda_{j}$ (see Section 5). In general, this condition on $Q_{\Omega}$ or on $\tilde{Q}_{\Omega}$, can be a quite restrictive condition for the higer codimension case, and it holds for calibrated submanifolds only if these are necessarily totally geodesic (see Proposition 1.3). But it holds for certain kind of submanifolds, as for example, if $F$ is sufficiently close to a totally umbilical submanifold (i.e. satisfy $B=H g$ ). For the Kähler calibration we will find in Proposition 5.3 that $\delta$-positiveness is quite unlike to hold on minimal 4-submanifolds with equal Kähler angles unless $\bar{M}$ is a Calabi-Yau 4-fold. In Lemma 5.5 for almost complex submanifolds in quaternionic Kähler manifolds, we give a natural condition that ensures $\delta$ positiveness of $Q_{\Omega}(B)$. 
For calibrated submanifolds we have:

Proposition 1.3. If $F: M \rightarrow \bar{M}$ is $\Omega$-calibrated then $Q_{\Omega}(B)=\tilde{Q}_{\Omega}(B)=0$. Thus, if $Q_{\Omega}$ is $\delta$-positive, then $F$ is totally geodesic. This is always the case $n=1$.

Theorem 1.5. Assume $M$ has parallel mean curvature, $\cos \theta>0$, and $Q_{\Omega}(B) \geq \delta\|B\|^{2}$ for some constant $\delta>0$ and

$$
\sum_{i j} \bar{R}\left(X_{i}, X_{j}, X_{i}, \Phi\left(X_{j}\right)\right) \geq 0 .
$$

Then the following inequalities hold for any compact domain D:

$$
\begin{gathered}
\|\nabla \log \cos \theta\| \leq \sqrt{m} \tan \theta\|B\|, \\
\int_{D}\|B\|^{2} d V \leq \frac{\sqrt{m}}{\delta} \int_{\partial D} \tan \theta\|B\| d A .
\end{gathered}
$$

Moreover:

(A) if $\tan \theta\|B\|$ is integrable on $M$ and $M$ is complete, then $F$ is totally geodesic;

(B) if $\inf _{M} \cos \theta=\tau>0$, and $\|B\|$ is not identically zero, then

$$
\frac{\inf _{M}\|B\|^{2}}{\sup _{M}\|B\|} \leq \inf _{D}\left(\frac{f_{D}\|B\|^{2} d V}{f_{\partial D}\|B\| d A}\right) \leq \frac{\sqrt{m}}{\delta} \frac{\sqrt{1-\tau^{2}}}{\tau} \mathfrak{h}(M)
$$

In particular, if $\|B\|$ is constant, then

$$
\|B\| \leq \frac{\sqrt{m}}{\delta} \frac{\sqrt{1-\tau^{2}}}{\tau} \mathfrak{h}(M) .
$$

In this case (since $\|B\| \neq 0), \mathfrak{h}(M) \neq 0$.

$(C)$ If the sectional curvatures of $\bar{M}$ are bounded from below, $M$ is complete, $\|B\|$ is bounded, then either $\inf _{M} \cos \theta=0$ or $\inf _{M}\|B\|=0$.

$\delta$-positiveness of $Q_{\Omega}(B)$ and (1.7) imply $\Delta \log \cos \theta \leq-\delta\|B\|^{2}$, and (A)-(C) are consequences of this. Parallel submanifolds (i.e. $\nabla B=0$ ) have parallel mean curvature, $\|B\|$ is constant, and equality to zero at (1.7). We will see in Section 5 that (1.7) holds for example for almost complex submanifolds in quaternionic space forms with nonnegative scalar curvature. If $n=1,(1.7)$ is equivalent to $\sum_{j} \operatorname{Ricci}^{\bar{M}}\left(X_{j}, \Phi\left(X_{j}\right)\right) \geq 0$, that holds for $\bar{M}$ an Einstein manifold.

For $M$ noncompact surface, using a criteria for parabolicity we prove next theorem: 
Theorem 1.6. Assume $F: M^{2} \rightarrow \bar{M}$ is a minimal complete immersed surface with $\cos \theta>0$, and $\bar{K} \circ F \geq 0$ away from a compact set of $M$, where $\bar{K}$ denotes the sectional curvatures of $\bar{M}$. If (1) or (2) below holds:

(1) $\bar{M}$ is a space form of dimension 3;

(2) $Q_{\Omega}(B) \geq \delta\|B\|^{2}, \cos \theta \geq \tau$, with $\tau, \delta>0$ constants and (1.7) holds;

then $M$ is a totally geodesic submanifold.

The previous theorem applied to graphs gives (simpler proofs) of the classical Bernstein results:

Corollary 1.3. (1) $[6,10]$ If a smooth entire function $f: \mathbb{R}^{2} \rightarrow \mathbb{R}$ defines a minimal graph in $\mathbb{R}^{3}$, then $f$ is linear.

(2) [37] If a smooth entire function $f: \mathbb{R}^{2} \rightarrow \mathbb{R}^{n}$ defines a minimal graph in $\mathbb{R}^{n+2}$, with $\left\|\wedge^{2} d f\right\|=\left|\lambda_{1} \lambda_{2}\right| \leq 1-\delta, 1 \geq \delta>0$ constant, and $\|d f\|$ bounded (or equivalently, $\cos \theta \geq \tau>0$ ), then $f$ is a linear map.

An application of Theorem 1.5 gives the following Bernstein-type results for graphic submanifolds:

Corollary 1.4. (3) Let $f:\left(M^{m}, g_{1}\right) \rightarrow\left(N^{n}, h\right)$ defining a minimal graph on $\bar{M}=M \times N$ with $\int_{M} \tan \theta\|B\| d V<+\infty$. We assume $\left(M, g_{1}\right)$ is complete with sectional curvature $K_{1}$ and Ricci tensor Ricci $i_{1}$ and $\left(N^{n}, h\right)$ has sectional curvature $K_{N}$ satisfying: (a) for $n=1$, Ricci $i_{1} \geq 0$; (b) for $n \geq 2, f^{*} h<$ $(1-\delta) g_{1}, 1 \geq \delta>0$, and at each $p \in M$ and two-planes $P$ of $T_{p} M$, and $P^{\prime}$ of $T_{f(p)} N$, either $K_{1}(P) \geq K_{N}\left(P^{\prime}\right)^{+}$, or $\operatorname{Ricci}_{1}(p) \geq 0$ and $K_{N}\left(P^{\prime}\right) \leq-K_{1}(P)$. Then $f$ is totally geodesic. Furthermore if at some point $K_{1}(p)>0$ (or Ricci $\left._{1}>0\right)$ then $f$ is constant, and $\Gamma_{f}$ is a slice.

For immersed hypersurfaces in the Euclidean space we have:

Corollary 1.5. Assume $M$ is a complete minimal immersed hypersurface of $\mathbb{R}^{m+1}$, such that for some parallel calibration $\Omega$ of $\mathbb{R}^{m+1}$, we have $\cos \theta>0$ on all $M$ (and so, $M$ is locally a graph). If $\int_{M} \sin \theta\|B\| d V<+\infty$, then $M$ is a linear hyperplane.

In Section 2 we derive the fundamental properties of the morphism $\Phi$ and prove Theorem 1.2 and Corollary 1.2. In Section 3 we discuss when $F$ is totally geodesic, describe the formula of the Laplacian of $\cos \theta$ and give the proof of Proposition 1.3, Theorems 1.5 and 1.6, and Corollaries 1.3, 1.4 and 1.5. In Section 4 we obtain some properties of the Cheeger constant, and prove Theorem 1.4 and Proposition 1.1. In Section 5 we specify to some examples of submanifolds in calibrated Riemannian manifolds, namely in a foliated space, in Kähler and quaternionic-Kähler manifolds. 


\section{The morphism $\Phi$}

Given $\Omega$ a $m$-calibration on $\bar{M}$, we consider the $T \bar{M}$-valued $(m-1)$-form $\Omega^{\sharp}: \wedge^{m-1} T \bar{M}^{*} \rightarrow T \bar{M}, \bar{g}\left(\Omega^{\sharp}\left(X_{2} \ldots, X_{m}\right), X_{1}\right)=\Omega\left(X_{1}, \ldots, X_{m}\right)$, where $X_{i} \in T_{p} \bar{M}$. Let $F: M \rightarrow \bar{M}$ be an immersed submanifold of dimension $m$ with normal bundle $N M$, and $\Omega$-angle $\theta$. We denote by $\bar{\nabla}, \nabla$ and $\nabla^{\perp}$ the respective covariant derivatives of $\bar{M}, M$ and $N M$, and by $B(X, Y)=\nabla_{X} d F(Y)$ the second fundamental form of $F$, defined by the following equations for $X, Y$ vector fields on $M$ and $U$ section of $N M$

$$
\nabla_{X} Y=\left(\bar{\nabla}_{X} Y\right)^{\top}, \quad\left(\bar{\nabla}_{X} Y\right)^{\perp}=B(X, Y), \quad \nabla_{X}^{\perp} U=\left(\bar{\nabla}_{X} U\right)^{\perp},
$$

where $(\cdot)^{\top}$ and $(\cdot)^{\perp}$ are the orthogonal projections into $T M$ and $N M$ respectively. The mean curvature is $H=\frac{1}{m}$ trace $B$. We consider the morphism $\Phi=\Phi_{\Omega}: T M \rightarrow N M$ defined in (1.3), $\Phi(X)=\left(\Omega^{\sharp}(* X)\right)^{\perp}$. Recall the covariant derivative and the co-differential of $\Phi$ are given by

$$
\nabla_{X} \Phi(Y)=\nabla_{X}^{\perp}(\Phi(Y))-\Phi\left(\nabla_{X} Y\right), \quad \delta \Phi=-\sum_{i} \nabla_{X_{i}} \Phi\left(X_{i}\right) .
$$

Lemma 2.1. If $X \in T_{p} M$ and $U \in N M_{p}$ are units, then $|\bar{g}(\Phi(X), U)| \leq$ $\sin \theta$.

Proof . Let $X_{i}$ be a direct o.n. basis of $T_{p} M$ with $X_{1}=X$. Consider the function

$$
\phi(t)=\Omega\left(\frac{\epsilon X_{1}+t U}{\sqrt{1+t^{2}}}, X_{2}, \ldots, X_{m}\right)=\frac{1}{\sqrt{1+t^{2}}}|\cos \theta|+\frac{t}{\sqrt{1+t^{2}}} \bar{g}(\Phi(X), U)
$$

where $\epsilon= \pm 1$ s.t. $\epsilon \cos \theta=|\cos \theta|$.

Since $\Omega$ is a calibration, $\phi(t) \leq 1$ for any $t$. We may assume $\cos \theta \neq 0$. At $t=\frac{\bar{g}(\Phi(X), U)}{|\cos \theta|}, \phi(t)=\sqrt{\cos ^{2} \theta+\bar{g}(\Phi(X), U)^{2}} \leq 1$.

Lemma 2.2. For $X_{i}$ and $U_{\alpha}$ d.o.n. basis of $T_{p} M$ and $N M_{p}$ respectively, we have

$$
\delta \Phi=m \cos \theta H-\sum_{\alpha}\left(\bar{\nabla}_{U_{\alpha}} \Omega\right)\left(X_{1}, \ldots, X_{m}\right) U_{\alpha}
$$

$$
\langle\nabla \Phi, B\rangle=-\cos \theta \tilde{Q}_{\Omega}(B)+\sum_{j k}\left(\bar{\nabla}_{X_{j}} \Omega\right)\left(X_{i}, \ldots, B\left(X_{j}, X_{k}\right)_{(k)}, \ldots, X_{m}\right)
$$

Proof. Let $U$ a section of $N M$ and $Z \in T_{p} M$. We may assume $\nabla^{\perp} U(p)=$ $\nabla X_{i}(p)=0$. At $p, g\left(\left(\bar{\nabla}_{Z} U\right)^{\top}, X\right)=-\bar{g}(B(Z, X), U), \bar{\nabla}_{Z} X_{i}(p)=\left(\bar{\nabla}_{Z} X_{i}(p)\right)^{\perp}=$ $B\left(Z, X_{i}\right)$, and

$$
\begin{aligned}
\bar{g}\left(\nabla_{Z} \Phi\left(X_{1}\right), U\right)= & Z \cdot \bar{g}\left(\Phi\left(X_{1}\right), U\right)=d\left(\Omega\left(U, X_{2}, \ldots, X_{m}\right)\right)(Z) \\
= & \bar{\nabla}_{Z} \Omega\left(U, X_{2}, \ldots, X_{m}\right)+\Omega\left(\left(\bar{\nabla}_{Z} U\right), X_{2}, \ldots, X_{m}\right) \\
& +\sum_{i \geq 2} \Omega\left(U, X_{2}, \ldots, \bar{\nabla}_{Z} X_{i}, \ldots, X_{m}\right) \\
= & \bar{\nabla}_{Z} \Omega\left(U, X_{2}, \ldots, X_{m}\right)+\Omega\left(\left(\bar{\nabla}_{Z} U\right)^{\top}, X_{2}, \ldots, X_{m}\right) \\
& +\sum_{i \geq 2} \bar{g}\left(\Omega^{\sharp}\left(X_{2}, \ldots, B\left(Z, X_{i}\right), \ldots, X_{m}\right), U\right) .
\end{aligned}
$$


That is

$$
\begin{aligned}
\nabla_{Z} \Phi\left(X_{1}\right)= & \sum_{\alpha} \bar{\nabla}_{Z} \Omega\left(U_{\alpha}, X_{2}, \ldots, X_{m}\right) U_{\alpha}-\cos \theta B\left(Z, X_{1}\right) \\
& +\sum_{i \geq 2}\left(\Omega^{\sharp}\left(X_{2}, \ldots, B\left(Z, X_{i}\right), \ldots, X_{m}\right)\right)^{\perp} .
\end{aligned}
$$

We have $* X_{k}=(-1)^{k-1} X_{1} \wedge \ldots \wedge \hat{X}_{k} \wedge \ldots \wedge X_{m}$. Hence,

$$
\begin{aligned}
\nabla_{Z} \Phi\left(X_{k}\right)= & \sum_{\alpha}(-1)^{k+1} \bar{\nabla}_{Z} \Omega\left(U_{\alpha}, X_{1}, \ldots, \hat{X}_{k}, \ldots X_{m}\right) U_{\alpha}-\cos \theta B\left(Z, X_{k}\right) \\
& +\sum_{1 \leq i<k}(-1)^{k+1}\left(\Omega^{\sharp}\left(X_{1}, \ldots, B\left(Z, X_{i}\right), \ldots, \hat{X}_{k}, \ldots, X_{m}\right)\right)^{\perp} \\
& +\sum_{k<i}(-1)^{k+1}\left(\Omega^{\sharp}\left(X_{1}, \ldots, \hat{X}_{k}, \ldots, B\left(Z, X_{i}\right), \ldots, X_{m}\right)\right)^{\perp} .
\end{aligned}
$$

Therefore,

$$
\begin{aligned}
\sum_{k} \nabla_{X_{k}} & \Phi\left(X_{k}\right)=\sum_{\alpha}-d \Omega\left(U_{\alpha}, X_{1}, \ldots, X_{m}\right) U_{\alpha} \\
& +\sum_{\alpha}\left(\bar{\nabla}_{U_{\alpha}} \Omega\right)\left(X_{1}, \ldots, X_{m}\right) U_{\alpha}-\sum_{k} \cos \theta B\left(X_{k}, X_{k}\right) \\
& +\sum_{k} \sum_{i<k}(-1)^{k+i}\left(\Omega^{\sharp}\left(B\left(X_{k}, X_{i}\right), X_{1}, \ldots, \hat{X}_{i}, \ldots, \hat{X}_{k}, \ldots, X_{m}\right)\right)^{\perp} \\
& +\sum_{k} \sum_{k<i}(-1)^{k+i-1}\left(\Omega^{\sharp}\left(B\left(X_{k}, X_{i}\right), X_{1}, \ldots, \hat{X}_{k}, \ldots, \hat{X}_{i}, \ldots, X_{m}\right)\right)^{\perp} .
\end{aligned}
$$

Interchanging $i$ by $k$ in the later line and using the symmetry of $B$ and that $d \Omega=0$ we prove the first equality of the lemma. The computation of the second equality is similar with $\langle\nabla \Phi, B\rangle=\sum_{j k} \bar{g}\left(\nabla_{X_{j}} \Phi\left(X_{k}\right), B\left(X_{j}, X_{k}\right)\right)$, recalling the definition in (1.6), where $\left\langle\Psi, \wedge^{2} B\right\rangle=\sum_{i<k} \sum_{j}\left\langle\Psi\left(X_{i}, X_{k}\right), B\left(X_{j}, X_{i}\right) \wedge\right.$ $\left.B\left(X_{j}, X_{k}\right)\right\rangle$.

Proof of Theorem 1.2. Consider the vector field $Z$ on $M$ defined by

$$
g(Z, X)=\bar{g}(\Phi(X), H) \quad \forall X \in T M .
$$

Using Lemma 2.2 we have

$$
\begin{aligned}
\operatorname{div}(Z) & =-\bar{g}(\delta \Phi, H)+\sum_{i} \bar{g}\left(\Phi\left(X_{i}\right), \nabla_{X_{i}}^{\perp} H\right) \\
& =-m \cos \theta\|H\|^{2}+\left\langle\nabla^{\perp} H, \Phi\right\rangle+\bar{\nabla}_{H} \Omega\left(X_{1}, \ldots, X_{m}\right) .
\end{aligned}
$$

Assume $Z \neq 0$. Take $X=Z /\|Z\|$ and $U=\Phi(X) /\|\Phi(X)\|$. By Lemma 2.1, $\|\Phi(X)\|^{2}=\bar{g}(\Phi(X), U)^{2} \leq \sin ^{2} \theta$. From this inequality and applying Schwartz inequality in $(2.1)$ we get

$$
\|Z\| \leq \sin \theta\|H\|
$$

If $\nu$ denotes the outward unit of $\partial D$, integration of (2.2) on $D$ and (2.3) gives

$$
\begin{aligned}
& \mid \int_{D}\left(-m \cos \theta\|H\|^{2}+\left\langle\nabla^{\perp} H,\right.\right.\Phi\rangle) d V+\int_{D} \bar{\nabla}_{H} \Omega \mid \\
& \leq\left|\int_{\partial D}\langle Z, \nu\rangle d A\right| \leq \int_{\partial D} \sin \theta\|H\| d A
\end{aligned}
$$


Theorem 1.3 and its Corollary 1.1 are an immediate consequence of Theorem 1.2.

Proof of Corollary 1.2. By Theorem 1.2,

$$
m \cos \theta \int_{M}\|H\|^{2} d V=\int_{M}\left\langle\nabla^{\perp} H, \Phi\right\rangle d V .
$$

We use Schwartz and a geometric-arithmetic inequality to obtain

$$
\begin{aligned}
\left|\left\langle\nabla^{\perp} H, \Phi\right\rangle\right| & \leq \sum_{i}\left|\bar{g}\left(\nabla_{X_{i}}^{\perp} H, \Phi\left(X_{i}\right)\right)\right| \\
& \leq \sum_{i}\left\|\nabla_{X_{i}}^{\perp} H\right\| \mu \sin \theta \leq \sqrt{m} \mu \sin \theta\left\|\nabla^{\perp} H\right\|,
\end{aligned}
$$

where $X_{i}$ is any orthonormal basis of $T_{p} M$.

If equality holds, then $\Phi\left(X_{i}\right)=\alpha_{i} \nabla_{X_{i}}^{\perp} H$ or $\nabla_{X_{i}}^{\perp} H=\beta_{i} \Phi\left(X_{i}\right)$, and if $\nabla_{X_{i}}^{\perp} H \neq 0$ we must have $\left\|\Phi\left(X_{i}\right)\right\|=\mu \sin \theta$, where $\alpha_{i}, \beta_{i} \in \mathbb{R}$. Since $\mu \sin \theta \neq 0$ we must have $\nabla_{X_{i}}^{\perp} H=\Phi\left(\psi\left(X_{i}\right)\right), \forall i$.

\section{3. $\Delta \cos \theta$}

In this section we are assuming $\Omega$ is parallel and $F: M^{m} \rightarrow \bar{M}^{m+n}$ is an immersion with $\cos \theta>0$. We use the curvature sign convention $\bar{R}(X, Y)=$ $-\left[\bar{\nabla}_{X}, \bar{\nabla}_{Y}\right]+\bar{\nabla}_{[X, Y]}$. Thus, $\bar{R}(X, Y, Z, W)=\bar{g}(\bar{R}(X, Y) Z, W)$.

Lemma 3.1. $\nabla \cos \theta=B_{\Phi}^{\sharp}$ and

$$
\begin{aligned}
\Delta \cos \theta & =-\cos \theta \tilde{Q}(B)+m\left\langle\nabla^{\perp} H, \Phi\right\rangle-\sum_{i j} \bar{R}\left(X_{i}, X_{j}, X_{i}, \Phi\left(X_{j}\right)\right) \\
\Delta \log (\cos \theta) & =-Q_{\Omega}(B)+\frac{m}{\cos \theta}\left\langle\nabla^{\perp} H, \Phi\right\rangle-\frac{1}{\cos \theta} \sum_{i j} \bar{R}\left(X_{i}, X_{j}, X_{i}, \Phi\left(X_{j}\right)\right) \\
\Delta\left(\frac{1}{\cos \theta}\right) & =\frac{1}{\cos \theta}\left(\hat{Q}_{\Omega}(B)-\frac{m}{\cos \theta}\left\langle\nabla^{\perp} H, \Phi\right\rangle+\frac{1}{\cos \theta} \sum_{i j} \bar{R}\left(X_{i}, X_{j}, X_{i}, \Phi\left(X_{j}\right)\right)\right)
\end{aligned}
$$

where $\hat{Q}_{\Omega}(B)=Q_{\Omega}(B)+\frac{1}{\cos ^{2} \theta}\left\|B_{\Phi}\right\|^{2}$. Moreover,

$$
\|\nabla \cos \theta\|^{2} \leq m \sin ^{2} \theta\|B\|^{2} \quad \text { and } \quad\|\Phi\|^{2} \leq m \sin ^{2} \theta .
$$

Furthermore, if $n=1,\|\Phi\|^{2} \geq \sin ^{2} \theta$. In the later case if $m=2$ and $H=0$, then

$$
\|\nabla \cos \theta\|^{2}=\frac{1}{2}\|B\|^{2}\left(\|\Omega\|^{2}-\cos ^{2} \theta\right)=\frac{1}{2}\|B\|^{2}\|\Phi\|^{2} .
$$

Remark. In this lemma the expression of $\Delta \cos \theta$ is still valid for $\cos \theta$ with any value in $\mathbb{R}$, since, at points where $\cos \theta=0, \cos \theta \tilde{Q}_{\Omega}(B)$ means $2\left\langle\Psi, \wedge^{2} B\right\rangle$. 
Proof. For a local orthonormal frame $X_{i}$, and where $(j)$ denotes "place $j$ ",

$$
\begin{aligned}
& d \cos \theta\left(X_{k}\right)=\sum_{j} \Omega\left(X_{1}, \ldots, \bar{\nabla}_{X_{k}} X_{j_{(j)}}, \ldots, X_{m}\right) \\
& \quad=\sum_{j} \Omega\left(X_{1}, \ldots, B\left(X_{k}, X_{j}\right)_{(j)}, \ldots, X_{m}\right)+\sum_{j} \Omega\left(X_{1}, \ldots, \nabla_{X_{k}} X_{j_{(j)}}, \ldots, X_{m}\right) \\
& \quad=\sum_{j} \Omega\left(X_{1}, \ldots, B\left(X_{k}, X_{j}\right)_{(j)}, \ldots, X_{m}\right)=B_{\Phi}\left(X_{k}\right),
\end{aligned}
$$

where we used in the last equality $\left\langle\nabla_{X_{k}} X_{j}, X_{j}\right\rangle=0$, and $\Omega\left(X_{1}, \ldots, X_{k_{(j)}}, \ldots\right.$, $\left.X_{m}\right)$ vanish for $k \neq j$. Differentiation of the later equation at $p$, and using that

$$
\begin{aligned}
\bar{\nabla}_{X_{k}} B\left(X_{k}, X_{j}\right) & =\nabla_{X_{k}} B\left(X_{k}, X_{j}\right)-\sum_{i} \bar{g}\left(B\left(X_{k}, X_{j}\right), B\left(X_{k}, X_{i}\right)\right) X_{i} \\
\sum_{k} \nabla_{X_{k}} B\left(X_{k}, X_{j}\right) & =\sum_{k} \nabla_{X_{k}} B\left(X_{j}, X_{k}\right)=m \nabla_{X_{j}}^{\perp} H-\sum_{k}\left(\bar{R}\left(X_{k}, X_{j}\right) X_{k}\right)^{\perp},
\end{aligned}
$$

where in the latter equality we used the Codazzi's equation for $B, \nabla_{X_{k}} B\left(X_{j}\right.$, $\left.X_{k}\right)=\nabla_{X_{j}} B\left(X_{k}, X_{k}\right)-\left(\bar{R}\left(X_{k}, X_{j}\right) X_{k}\right)^{\perp}$, we have

$\triangle \cos \theta=\sum_{k} \nabla_{X_{k}} d \cos \theta\left(X_{k}\right)$

$$
\begin{aligned}
& =\sum_{k} \sum_{s<j} 2 \Omega\left(X_{1}, \ldots, B\left(X_{s}, X_{k}\right)_{(s)}, \ldots, B\left(X_{j}, X_{k}\right)_{(j)}, \ldots, X_{m}\right) \\
& +\sum_{k j} \Omega\left(X_{1}, \ldots, \nabla_{X_{k}} B\left(X_{k}, X_{j}\right)_{(j)}, \ldots, X_{m}\right)-\cos \theta \bar{g}\left(B\left(X_{k}, X_{j}\right), B\left(X_{k}, X_{j}\right)\right) \\
& =\sum_{s<j} \sum_{k} 2\left\langle\Psi\left(X_{s}, X_{j}\right), B\left(X_{s}, X_{k}\right) \wedge B\left(X_{j}, X_{k}\right)\right\rangle+m\left\langle\nabla^{\perp} H, \Phi\right\rangle \\
& -\cos \theta\|B\|^{2}-\sum_{k j} \bar{R}\left(X_{k}, X_{j}, X_{k}, \Phi\left(X_{j}\right)\right) .
\end{aligned}
$$

The lemma now follows from the expressions of $\Delta \log \cos \theta$ and $\Delta\left(\frac{1}{\cos \theta}\right)$ in terms of $\Delta \cos \theta$. Next we estimate $\|\nabla \cos \theta\|^{2}$ and $\|\Phi\|$. By Lemma 2.1, $\left\|\Phi\left(X_{k}\right)\right\| \leq \sin \theta$, and so $\|\Phi\|^{2} \leq m \sin ^{2} \theta$. Now, from the first equation in this proof,

$$
\begin{aligned}
\|\nabla \cos \theta\|^{2} & =\left\|B_{\Phi}\right\|^{2} \leq \sum_{i j k} \sin ^{2} \theta\left\|B_{i j}\right\|\left\|B_{i k}\right\| \\
& \leq \sum_{i j k} \frac{\sin ^{2} \theta}{2}\left(\left\|B_{i j}\right\|^{2}+\left\|B_{i k}\right\|^{2}\right) \leq m \sin ^{2} \theta\|B\|^{2},
\end{aligned}
$$

where $B_{i j}=B\left(X_{i}, X_{j}\right)$. Note that $\|\Omega\|^{2} \geq 1$, because there exists a calibrated subspace. Consequently, if $n=1,1 \leq\|\Omega\|^{2}=\cos ^{2} \theta+\|\Phi\|^{2}$ what implies $\|\Phi\|^{2} \geq \sin ^{2} \theta$. Finally in case $m=2$ and $n=1$, let $\nu$ be a unit normal to $M$ and set $B_{i j}=\bar{g}\left(B\left(X_{i}, X_{j}\right), \nu\right)$. From minimality of $F$

$$
\begin{aligned}
\|\nabla \cos \theta\|^{2}= & \Omega\left(B\left(X_{1}, X_{1}\right), X_{2}\right)^{2}+\Omega\left(B\left(X_{2}, X_{1}\right), X_{1}\right)^{2} \\
& +\Omega\left(B\left(X_{1}, X_{2}\right), X_{2}\right)^{2}+\Omega\left(B\left(X_{2}, X_{2}\right), X_{1}\right)^{2} \\
= & B_{11}^{2} \Omega\left(\nu, X_{2}\right)^{2}+B_{12}^{2} \Omega\left(\nu, X_{1}\right)^{2}+B_{12}^{2} \Omega\left(\nu, X_{2}\right)^{2}+B_{22}^{2} \Omega\left(\nu, X_{1}\right)^{2} \\
= & \frac{1}{2}\|B\|^{2}\left(\Omega\left(\nu, X_{2}\right)^{2}+\Omega\left(\nu, X_{1}\right)^{2}\right)=\frac{1}{2}\|B\|^{2}\left(\|\Omega\|^{2}-\Omega\left(X_{1}, X_{2}\right)^{2}\right) .
\end{aligned}
$$


An immediate consequence from the last equality of Lemma 3.1 follows next:

Proposition 3.1. If $m=2$ and $n=1, F$ is minimal and $\cos \theta$ is constant, then either $F$ is a calibrated submanifold or it is totally geodesic.

Proposition 3.2. If $F$ is a parallel submanifold then $(1.7)=0$ holds.

Proof. From proof of Lemma 3.1

$$
\sum_{k} \nabla_{X_{k}} B\left(X_{k}, X_{j}\right)=m \nabla_{X_{j}} H-\sum_{k}\left(\bar{R}\left(X_{k}, X_{j}\right) X_{k}\right)^{\perp} .
$$

Since $\nabla B=0$, then $\sum_{k}\left(\bar{R}\left(X_{k}, X_{j}\right) X_{k}\right)^{\perp}=0$.

Proof of Proposition 1.3. Since $F$ is calibrated, $F$ is minimal, and by Lemma $2.1 \Phi=0$. From Lemma $3.1,0=Q_{\Omega}(B)=\tilde{Q}_{\Omega}(B)$, and so $B=0$.

Recall the average value of a function $f$ on a domain $D$ and on $\partial D$ is given by:

$$
f_{D} f d V=\frac{1}{V(D)} \int_{D} f d V, \quad f_{\partial D} f d A=\frac{1}{A(\partial D)} \int_{\partial D} f d A
$$

Proof of Theorem 1.5. Using Lemma 3.1 we obtain the first inequality of (1.8), and under the assumptions of the theorem we have

$$
\Delta \log \cos \theta \leq-Q_{\Omega}(B) \leq-\delta\|B\|^{2} .
$$

Thus, applying Lemmas 2.1 and 3.1, we have after integration of (3.1),

$$
\delta \int_{D}\|B\|^{2} \leq \int_{\partial D}-g(\nabla \log \cos \theta, \nu) d V \leq \int_{\partial D} \sqrt{m} \sup _{\partial D} \tan \theta\|B\| d A
$$

and second inequality of (1.8) is proved. If $\tan \theta\|B\|$ is integrable on $M$, then so it is $\nabla \log \cos \theta$, by the first inequality of (1.8). Since $-\log \cos \theta$ is a subharmonic function by (3.1), then applying the Stokes theorem for complete manifolds in the version given by Yau ([38] Corollary page 660), we conclude $\Delta \log \cos \theta=0$. From (3.1), this implies $B=0$ on $D$, and (A) is proved. (B) follows immediately. To prove $(\mathrm{C})$ we use the Omori-Cheng-Yau maximum principle. Under the assumptions, by Gauss equation $R i c c i^{M}$ is bounded from below. If we assume $\inf _{M} \cos \theta>0$, then we take a sequence $p_{k}$ such that $u\left(p_{k}\right) \rightarrow \sup _{M} u, \nabla u\left(p_{k}\right) \rightarrow 0$ and $\lim _{k} \Delta u\left(p_{k}\right) \leq 0$ when $k \rightarrow+\infty$, where $u=\log (\cos \theta)^{-1}$. This implies by Lemma 3.1 that $Q_{\Omega}(B)\left(x_{k}\right) \rightarrow 0$, and so $\inf _{M}\|B\|=0$. 
Proof of Theorem 1.6. Recall that if a surface is parabolic, any nonnegative superharmonic function is constant. By Gauss equation, the sectional curvature of $M$ satisfies $K_{M}=\bar{R}\left(X_{1}, X_{2}, X_{1}, X_{2}\right)-\frac{1}{2}\|B\|^{2}$. Let $C$ be a compact set of $M$ such that $\bar{K} \circ F \geq 0$ away from $C$. (1) Since $\bar{M}$ is 3dimensional, $Q_{\Omega}(B) \geq\|B\|^{2}$ and by Lemma $3.1,\|\nabla \cos \theta\|^{2} \geq \frac{1}{2}\|B\|^{2} \sin ^{2} \theta$. Again, by Lemma 3.1 and the assumptions of the theorem we have $\Delta \cos \theta \leq$ $-\cos \theta\|B\|^{2}$ and so

$$
\begin{aligned}
\Delta \log (1+\cos \theta) & =\frac{1}{(1+\cos \theta)}\left(\Delta \cos \theta-\frac{\|\nabla \cos \theta\|^{2}}{(1+\cos \theta)}\right) \\
& \leq \frac{1}{(1+\cos \theta)}\left(-\cos \theta\|B\|^{2}-\frac{\|\nabla \cos \theta\|^{2}}{(1+\cos \theta)}\right) \leq 0
\end{aligned}
$$

We consider on $M$ the complete metric $\tilde{g}=(1+\cos \theta)^{p} g$, where we choose $p \geq 2$. This metric has sectional curvature $\tilde{K}$ that satisfies on $M \sim C$

$$
\begin{aligned}
\tilde{K} & =\frac{1}{(1+\cos \theta)^{p}}\left(\bar{R}\left(X_{1}, X_{2}, X_{1}, X_{2}\right)-\frac{1}{2}\|B\|^{2}-\frac{p}{2} \Delta \log (1+\cos \theta)\right) \\
& \geq \frac{1}{(1+\cos \theta)^{p}}\left(-\frac{1}{2}\|B\|^{2}+\frac{p}{2} \frac{1}{(1+\cos \theta)}\left(\cos \theta\|B\|^{2}+\frac{\|\nabla \cos \theta\|^{2}}{(1+\cos \theta)}\right)\right) \\
& \geq \frac{1}{(1+\cos \theta)^{p}}\left(-\frac{1}{2}+\frac{p}{2} \frac{\cos \theta}{(1+\cos \theta)}+\frac{p}{4} \frac{\sin ^{2} \theta}{(1+\cos \theta)^{2}}\right)\|B\|^{2} \\
& =\frac{p-2}{4(1+\cos \theta)^{p}}\|B\|^{2} .
\end{aligned}
$$

Note that $d \tilde{V}=(1+\cos \theta)^{p} d V$ and so $\int_{M} \tilde{K}^{-} d \tilde{V}<+\infty$, where $\tilde{K}^{-}=$ $\max \{-\tilde{K}, 0\}$. This implies that $(M, \tilde{g})$ is parabolic, and so it is $(M, g)$, since $\tilde{\Delta}=(1+\cos \theta)^{-p} \Delta$. From (3.2) we have $\cos \theta$ constant and since $\cos \theta>0$, we conclude that $B=0$. (2) We consider on $M$ the metric $\tilde{g}=\cos ^{\frac{1}{\delta}} \theta g$. This metric is complete because $1 \geq \cos \theta \geq \tau>0$, and by Lemma 3.1 and the assumptions on the theorem, the sectional curvature satisfies on $M \sim C$,

$$
\tilde{K}=\cos ^{-\frac{1}{\delta}} \theta\left(K_{M}-\frac{1}{2 \delta} \Delta \log \cos \theta\right) \geq \cos ^{-\frac{1}{\delta}} \theta \bar{R}\left(X_{1}, X_{2}, X_{1}, X_{2}\right) \geq 0
$$

and $\Delta\left(\frac{1}{\cos \theta}-\frac{1}{\tau}\right) \geq \frac{\delta}{\cos \theta}\|B\|^{2}$. By the same arguments as in (1), $B=0$.

Proof of Corollaries 1.3 and 1.4. First we note that the graph $\Gamma_{f}$ of a map $f:\left(M, g_{1}\right) \rightarrow(N, h)$ defines a complete submanifold of $\left(M \times N, g_{1} \times h\right)$ provided $\left(M, g_{1}\right)$ is complete. We are considering $\Omega$ the volume calibration. (1) is a result of Theorem 1.6(1), since graphs satisfy $\cos \theta>0$. (2) is a consequence of Theorem 1.6(2), because the eigenvalues of $f^{*} h$ satisfy $\left|\lambda_{1} \lambda_{2}\right| \leq$ 
$1-\delta$, what implies $Q_{\Omega}(B) \geq \delta\|B\|^{2}$ and $\cos \theta \geq \tau=\left(1+C+(1-\delta)^{2}\right)^{-1 / 2}$, where $\|d f\|^{2} \leq C$ (see Subsection 5.1). To prove (3) we use Theorem 1.5 (A). Since $\lambda_{i}^{2} \leq 1-\delta$ (case $\left.n \geq 2\right), Q_{\Omega}$ is $\delta$-positive. Now we only have to check that (1.7) holds. Using $X_{i}$ and $X_{m+\alpha}$ suitable orthonormal frames of $\left(T M, g=g_{1}+f^{*} h\right)$ and of $(N M, \bar{g})$ respectively (see Section 5$)$, we have

$$
\begin{aligned}
& \sum_{i \neq j} \bar{R}\left(X_{i}, X_{j}, X_{i}, \Phi\left(X_{j}\right)\right)= \\
& =\cos \theta \sum_{i \neq j} \frac{\lambda_{j}^{2}}{\left(1+\lambda_{i}^{2}\right)\left(1+\lambda_{j}^{2}\right)}\left(R_{1}^{M}\left(a_{i}, a_{j}, a_{i}, a_{j}\right)\right. \\
& \left.\quad-\lambda_{i}^{2} R^{N}\left(a_{i+m}, a_{j+m}, a_{i+m}, a_{j+m}\right)\right) \\
& =\cos \theta \sum_{j} \sum_{i \neq j}\left(\frac{\lambda_{j}^{2}}{\left(1+\lambda_{j}^{2}\right)} K_{1}\left(a_{i}, a_{j}\right)\right. \\
& \left.\quad-\frac{\lambda_{i}^{2} \lambda_{j}^{2}}{\left(1+\lambda_{i}^{2}\right)\left(1+\lambda_{j}^{2}\right)}\left(K_{1}\left(a_{i}, a_{j}\right)+K_{N}\left(a_{i+m}, a_{j+m}\right)\right)\right) \\
& =\cos \theta\left(\sum_{j} \frac{\lambda_{j}^{2}}{\left(1+\lambda_{j}^{2}\right)} \operatorname{Ricci}_{1}\left(a_{j} a_{j}\right)\right. \\
& \left.\quad-\sum_{j \neq i} \frac{\lambda_{i}^{2} \lambda_{j}^{2}}{\left(1+\lambda_{i}^{2}\right)\left(1+\lambda_{j}^{2}\right)}\left(K_{1}\left(a_{i}, a_{j}\right)+K_{N}\left(a_{i+m}, a_{j+m}\right)\right)\right)
\end{aligned}
$$

If $n=1, \lambda_{i}=0$ for $i \geq 2$ and the last term of (3.4) disappears. For $n \geq 2$, if we assume at each point $p \in M$ and two-planes $P$ of $T_{p} M$ and $P^{\prime}$ of $T_{f(p)} N, K_{1}(P) \geq K_{N}\left(P^{\prime}\right)^{+}=\max \left\{K_{N}\left(P^{\prime}\right), 0\right\}$ (or Ricci $i_{1}(p) \geq 0$ and, $K_{N}\left(P^{\prime}\right) \leq-K_{1}(P)$ respectively) then we get

$$
\sum_{i \neq j} \bar{R}\left(X_{i}, X_{j}, X_{i}, \Phi\left(X_{j}\right)\right) \geq 0
$$

from (3.3) (from (3.4) respectively). This implies by Theorem 1.5, that $\Gamma_{f}$ is a totally geodesic submanifold of $\bar{M}$, on therefore $f:\left(M, g_{1}\right) \rightarrow(N, h)$ is also totally geodesic (see [30] or [25, 32]), and so we have equality to zero in all above equalities (see Proposition 3.2). In this case all $\lambda_{i}$ are constant. Moreover if at some point $K_{1}(p)>0$ (or $\operatorname{Ricci}_{1}>0$ respectively) then $\lambda_{j}=0$ for all $j$, and $f$ is constant.

Proof of corollary 1.5. We have $\cos \theta>0, \tilde{Q}_{\Omega}(B)=Q_{\Omega}(B) \geq\|B\|^{2}$ and (1.7) holds as well. In this case we use a modified version of the proof of Theorem $1.5(\mathrm{~A})$, by considering $\Delta \cos \theta=-\cos \theta \tilde{Q}_{\Omega}(B) \leq 0$. Integrability of $\|\nabla \cos \theta\| \leq \sin \theta\|B\|$ implies $\Delta \cos \theta=0$, and so $\|B\|=0$, that is $M$ is a hyperplane. 


\section{The Cheeger constant of a submanifold}

In this section we estimate the Cheeger constant (1.1) of a Riemannian manifold $(M, g)$. If $\partial M \neq \emptyset$ then $D$ satisfies $\partial D \cap \partial M=\emptyset$. If $M$ closed we may let $D=M$.

Proposition 4.1. [39] If $M$ is complete simply connected and the sectional curvature satisfies $K_{M} \leq-K, K$ a positive constant, then

$$
\mathfrak{h}(M) \geq(m-1) \sqrt{K}
$$

The proof is based on the use of the comparison theorem to obtain $\Delta r \geq$ $(m-1) \sqrt{K}$ where $r$ is the distance function to a point, and integration on a domain $D$.

Proposition 4.2. If $M$ is complete and Ricci ${ }^{M} \geq 0$ then $\mathfrak{h}(M)=0$.

Proof. If we assume the Ricci curvature of $M$ satisfies $\operatorname{Ricci}^{M} \geq 0$, following [3] for $m=2$, by a result due to Cheng [8] the first eigenvalue of the Dirichlet problem on a geodesic ball $B_{r}(p)$ is less than or equal to the first eigenvalue of a geodesic ball of the same radius of $\mathbb{R}^{m}$, that is $C_{1} / r^{2}$ for some constant $C_{1}>0$ that does not depend on $r$. Therefore $\lambda_{1}\left(B_{r}(p)\right) \leq C_{1} / r^{2}$, for $0<r<+\infty$. By a well known inequality due to Cheeger (Theorem 3 p. 95 in [7]), we get $\mathfrak{h}^{2}(M) \leq \mathfrak{h}^{2}\left(B_{r}(p)\right) \leq 4 \lambda_{1}\left(B_{r}(p)\right) \leq \frac{4 C_{1}}{r^{2}}$. This implies for $M$ complete that $\mathfrak{h}(M)=0$.

Given a smooth function $f: M \rightarrow \mathbb{R}$ and a regular value of $|f|, t \in R_{|f|}$, the sets

$$
\begin{gathered}
D_{f}^{+}(t)=\{p \in M:|f(p)| \geq t\}, \quad D_{f}^{-}(t)=\{p \in M:|f(p)| \leq t\}, \\
\Sigma_{f}(t)=\{p \in M:|f(p)|=t\}
\end{gathered}
$$

define smooth submanifolds with $\partial D_{f}^{ \pm}(t)=\Sigma_{f}(t)$. Set $V_{ \pm}(t)=V\left(D_{f}^{ \pm}(t)\right)$ and $A(t)=A\left(\Sigma_{f}(t)\right)$. Then $V_{ \pm}(t)$ are smooth on $R_{|f|}$ and the co-area formula (see e.g. [7]) states that for any nonnegative mensurable function $h$ (or $\left.h \in L^{1}(M)\right)$,

$$
\int_{M} h\|\nabla f\| d V=\int_{0}^{+\infty} d t \int_{\Sigma_{f}(t)} h d A(t) .
$$

Applying the co-area formula to $h=\|\nabla f\|^{-1} \mathcal{X}_{A}$, with $A=D_{f}^{\mp}(s)$ for $s$ regular value, where $\mathcal{X}_{A}$ is the characteristic function of a set $A$, one obtains

$$
V_{\mp}^{\prime}(s)= \pm \int_{\Sigma_{f}(s)}\|\nabla f\|^{-1} d A(s) .
$$


Lemma 4.1. If $D_{f}^{\mp}(s)$ is compact for $s<\sup |f| \quad(s>\inf |f|$ resp. $)$, then

$$
\mathfrak{h}(M) \leq \pm \frac{f_{D_{f}^{\mp}(s)}\|\nabla f\| d V}{s-f_{D_{f}^{\mp}(s)}|f| d V}
$$

Proof. Using the co-area formulas for $f$ restricted to the interior of $D_{f}^{-}(s)$ (at regular values $s$ ), and that $\mathfrak{h}(M) \leq A(t) / V_{-}(t), \forall t<s$, we have

$$
\begin{aligned}
\int_{D_{f}^{-}(s)}\|\nabla f\| d V & =\int_{0}^{s} A(t) d t \geq \int_{0}^{s} \mathfrak{h}(M) V_{-}(t) d t \\
& \left.=\mathfrak{h}(M)\left(t V_{-}(t)\right]_{0}^{s}-\int_{0}^{s} t V_{-}^{\prime}(t)\right) \\
& =\mathfrak{h}(M)\left(s V_{-}(s)-\int_{0}^{s} \int_{\Sigma_{f}(t)}|f(x)|\|\nabla f\|^{-1} d A(t) d t\right) \\
& =\mathfrak{h}(M)\left(s V_{-}(s)-\int_{D_{f}^{-}(s)}|f(x)| d V\right)
\end{aligned}
$$

where in the last equality we use the co-area formula for $h=\frac{|f|}{\|\nabla f\|}$. Similarly for $D_{f}^{+}(s)$. Note that the functions

$$
s-f_{D_{f}^{-}(s)}|f| d V=\int_{0}^{s} V_{-}(t) d t \quad \text { and } \quad-s+f_{D_{f}^{+}(s)}|f| d V=\int_{s}^{t_{+}} V_{+}(t) d t
$$

where $t_{+}=\sup _{M}|f|$, are increasing on $s$.

Corollary 4.1. The Cheeger constant of $M$ vanish if there exist a smooth function $f$ such that $D_{f}^{-}(s)$ is compact $\forall s<\sup _{M}|f|=+\infty$, and for some constants $0<\alpha, \delta<1$, we have $f_{D_{f}^{-}(s)}\|\nabla f\| d V \leq s^{\alpha}$, and $f_{D_{f}^{-}(s)}|f| \leq$ $(1-\delta) s$, when $s \rightarrow+\infty$.

Let $r$ be the distance function to a point $p$. Recalling that $\|\nabla r\|=1$, we have

Corollary 4.2. If $r^{2}$ is smooth for $r<s$, then

$$
\mathfrak{h}(M) \leq f_{B_{s}(p)} 2 r d V /\left(s^{2}-f_{B_{s}(p)} r^{2} d V\right) .
$$

In particular, if $f_{B_{s}(p)} r^{2} d V \leq(1-\delta) s^{2}$, where $0<\delta<1$ is a constant, $(M, g)$ is complete, and $r^{2}$ smooth on $M$, then $\mathfrak{h}(M)=0$. 
Note that in the previous corollary,

$$
f_{B_{s}(p)} r d V \leq\left(f_{B_{s}(p)} r^{2} d V\right)^{1 / 2} \leq \sqrt{(1-\delta)} s
$$

and $D_{f}^{-}\left(s^{2}\right)=B_{s}(p)$. Thus, if $\mathfrak{h}(M) \neq 0$, then $\lim _{s \rightarrow+\infty} \frac{1}{s^{2}} f_{B_{s}(p)} r^{2} d V=1$. If $M=\mathbb{R}^{m}$, in corollary 4.2 we may take $\delta=\frac{m}{2+m}$.

If $M$ is a submanifold of a $(m+n)$-dimensional Riemannian manifold $(\bar{M}, \bar{g})$ we can also estimate the Cheeger constant of $M$ under certain conditions. Recall $([29,28])$ that a vector field $\bar{X}$ on $\bar{M}$ is strongly convex on a open set $U$ of $\bar{M}$ if

$$
L_{\bar{X}} \bar{g} \geq 2 \alpha \bar{g}
$$

where $\alpha>0$ is a constant. Examples of such vector fields are $\frac{1}{2} \bar{\nabla} \bar{r}^{2}=r \frac{\partial}{\partial r}$ on a geodesic ball of $\bar{M}$ of radius $R$ and center $\bar{p}$ that does not intercept the cut locus at $\bar{p}$ and $\sqrt{\kappa} R<\pi / 2$ where $\kappa=\max \left\{0, \sup _{B_{R}(\bar{p})} \bar{K}\right\}$ and $\bar{K}$ are the sectional curvatures of $\bar{M}$. A strictly convex function $f$ on $\bar{M}$ with Hess $f \geq \alpha \bar{g}$ defines a strongly convex vector field $\nabla f$. Positive homothetic no-Killing vector fields are strongly convex. In $\mathbb{R}^{m+n}$ the position vector field $\bar{X}_{x}=x$ is such an example. If $F: M \rightarrow \bar{M}$ is an immersed submanifold let $X_{F}$ denote the vector field $X$ along $F$.

Lemma 4.2. If $\bar{M}$ carries a strongly convex vector field $\bar{X}$ on a neighbourhood of an immersion $F: M \rightarrow \bar{M}$ then

$$
\left(\sup _{M}\left\|\bar{X}_{F}\right\|\right)^{-1} \leq \frac{1}{\alpha}\left(\frac{1}{m} \mathfrak{h}(M)+\sup _{M}\|H\|\right) .
$$

In particular if $M$ is minimal and has zero Cheeger constant then $\bar{X}_{F}$ is unbounded.

Proof. By an elementary computation (see [21] or [29]) for any immersion $F$

$$
m \bar{g}\left(H, \bar{X}_{F}\right)=\operatorname{div}_{g}\left(\bar{X}_{F}^{T}\right)-\frac{1}{2} t r_{g} L_{\bar{X}} \bar{g}
$$

where $\bar{X}_{F}^{T}$ is the projection of $\bar{X}_{F}$ onto $T M$. Integration on a domain $D$ gives

$$
\begin{aligned}
\alpha m V(D) & \leq \int_{\partial D} g\left(\bar{X}_{F}^{T}, \nu\right) d A-\int_{D} m \bar{g}\left(H, \bar{X}_{F}\right) d V \\
& \leq \sup _{D}\left\|\bar{X}_{F}\right\|\left(A(\partial D)+\int_{D} m\|H\| d V\right)
\end{aligned}
$$

where $\nu$ is a unit normal to $\partial D$. Thus

$$
\left(\sup _{D}\left\|\bar{X}_{F}\right\|\right)^{-1} \leq \frac{1}{\alpha}\left(\frac{1}{m} \frac{A(\partial D)}{V(D)}+f_{D}\|H\| d V\right),
$$

with $f_{D}\|H\| d V \leq \sup _{M}\|H\|$. Taking the infimum on $D$ we obtain the proposition. 
Corollary 4.3. If $F: M \rightarrow \mathbb{R}^{n+m}$ is a minimal immersion with zero Cheeger constant, then $F$ is unbounded.

Proof of Theorem 1.4. Using Theorem 1.3, on each ball $B_{r}(p)$, and for any domain $D \subset B_{r}(p),\|H\| \leq \frac{1}{m}\left(\inf _{B_{r}(p)} \cos \theta\right)^{-1} \frac{A(\partial D)}{V(D)}$, and so, taking the infimum for $D \subset B_{r}(p), m\|H\| \leq\left(\inf _{B_{r}(p)} \cos \theta\right)^{-1} \mathfrak{h}\left(B_{r}(p)\right)$. By assumption of the theorem $\inf _{B_{r}(p)} \cos \theta \geq C r^{-\beta}$, and (1.4) leads to $\|H\| \leq C r^{\beta-1}$ for some constant $C>0$ that does not depend on $r$. Thus, letting $r \rightarrow+\infty$ we obtain $\|H\|=0$, and theorem 1.4 is proved.

Proposition 1.1 is an immediate consequence of Proposition 4.2 and Theorem 1.1.

If under certain conditions we have $\Delta \cos \theta \leq 0$, (as in Theorem 1.5) and $\theta$ is not constant, by the maximum principle, for any regular value of $\cos \theta, D^{-}(\epsilon)=\{p: \cos \theta \leq \epsilon\}$ cannot be a compact domain. Next we assume for $\epsilon>0$, the set $D^{+}(\epsilon)=\{p: \cos \theta \geq \epsilon\}$ to be compact. The next proposition is an attempt to understand what happens if one replaces the assumption $Q(B) \geq \delta\|B\|^{2}$ by the weaker condition $Q(B) \geq 0$. .

Proposition 4.3. Assume $\cos \theta>0$, and $D^{+}(\epsilon)$ is compact $\forall \epsilon \in(0,1]$. Then:

(a) If there exists constants $\alpha, \delta \in(0,1)$ such that

$f_{D^{+}(\epsilon)}(\cos \theta)^{-1} d V \leq(1-\delta) \frac{1}{\epsilon}$ and $f_{D^{+}(\epsilon)} \sin \theta \cos \theta^{-2}\|B\| \leq\left(\frac{1}{\epsilon}\right)^{\alpha}$, for $\epsilon \rightarrow 0$,

then $\mathfrak{h}(M)=0$.

(b) If $M$ is immersed with parallel mean curvature,

$$
Q_{\Omega}(B)+(\cos \theta)^{-2}\left\|B_{\Phi}\right\|^{2} \geq 0
$$

and (1.7) holds, and for some constant $\alpha>1$,

$$
\int_{M}(\cos \theta)^{-(\alpha+2)} \sin \theta\|\bar{R}\| d V<+\infty
$$

then

$$
\text { either } \int_{M}(\cos \theta)^{-(\alpha+4)}\|B\|^{2} d V=+\infty, \quad \text { or } M \text { is compact. }
$$

Proof. By Lemma 3.1, $f=1 / \cos \theta$ in (a) satisfies $f_{D^{+}(\epsilon)}\|\nabla f\| \leq\left(\frac{1}{\epsilon}\right)^{\alpha}$, and in (b) $\Delta f \geq 0$ and $f>0$. Then (a) follows from Corollary 4.1. Now 
we prove (b). For each $s$ fixed we consider the compact sets $D_{f}^{-}(s)$ and $\Sigma_{f}(s)$ and follow close the proof in [26, Lemma 7.1] replacing $r$ by $f$. We take a cut off function $\phi(f): M \rightarrow \mathbb{R}_{0}^{+}$where $\phi: \mathbb{R}_{0}^{+} \rightarrow[0,2]$ is a smooth nonnegative bounded function satisfying $\phi(t)=1$ if $t \leq s, \phi(t)=0$ if $t \geq 2 s$, and $\left(\phi^{\prime}\right)^{2} \leq C s^{-2}$ and $\left|\phi^{\prime \prime}\right| \leq C s^{-2}, C>0$ a constant that does not depend on $s$ (see for example, [26, Lemmas 7.1 and 6.1] and [38, p. 661]). Assume $2 s \in R_{f}$. Then integrating $\Delta\left(\phi^{2}(f) f^{\alpha-1} f\right)$ on $D_{f}^{-}(2 s)$, applying Stokes and using that $\Delta f^{\alpha-1} \geq 0$ we have

$$
\begin{aligned}
0 & \leq \int_{D_{f}^{-}(2 s)} \phi^{2}(f) f^{\alpha-1} \Delta f d V \\
\leq & \int_{D_{f}^{-}(2 s)}-\left(2 \phi(f) \Delta(\phi(f))+2\|\nabla(\phi(f))\|^{2}\right) f^{\alpha} d V \\
& -\int_{D_{f}^{-}(2 s)}\left(4(\alpha-1) \phi(f)\langle\nabla(\phi(f)), \nabla f\rangle f^{\alpha-1}+2\left\langle\nabla\left(\phi^{2}(f) f^{\alpha-1}\right), \nabla f\right\rangle\right) d V .
\end{aligned}
$$

Hence,

$$
\begin{aligned}
& 2 \int_{D_{f}^{-}(2 s)} \phi^{2}(f)(\alpha-1) f^{\alpha-2}\|\nabla f\|^{2} \leq \\
& \leq \int_{D_{f}^{-}(2 s)}-2 \phi(f)\left(\phi^{\prime \prime}(f)\|\nabla f\|^{2}+\phi^{\prime}(f) \Delta f\right) f^{\alpha} d V \\
&-\int_{D_{f}^{-}(2 s)} 2\left(\|\nabla(\phi(f))\|^{2} f^{\alpha}+2(\alpha-1) \phi(f)\langle\nabla(\phi(f)), \nabla f\rangle f^{\alpha-1}\right) d V \\
&-4 \int_{D_{f}^{-}(2 s)} \phi(f)\langle\nabla(\phi(f)), \nabla f\rangle f^{\alpha-1} d V \\
& \leq \int_{D_{f}^{-}(2 s)} \frac{\tilde{C}}{s^{2}}\|\nabla f\|^{2} f^{\alpha}+\frac{\tilde{C}}{s} \Delta f f^{\alpha}+(\alpha-1) \frac{\tilde{C}}{s} f^{\alpha-1}\|\nabla f\|^{2} \\
&+\int_{D_{f}^{-}(2 s)}-4 \phi(f) f^{\alpha-1}\langle\nabla(\phi(f)), \nabla f\rangle,
\end{aligned}
$$

where $\tilde{C}>0$ denotes a constant that does not depend on $s$. On the other hand

$$
\begin{aligned}
& -4 \phi(f) f^{\alpha-1}\langle\nabla(\phi(f)), \nabla f\rangle=-4\left\langle f^{\frac{\alpha}{2}} \nabla(\phi(f)), f^{\frac{\alpha-2}{2}} \phi(f) \nabla f\right\rangle \\
& \quad \leq 2\left(\frac{2}{(\alpha-1)}\|\nabla(\phi(f))\|^{2} f^{\alpha}+\frac{(\alpha-1)}{2} f^{\alpha-2} \phi(f)^{2}\|\nabla f\|^{2}\right) .
\end{aligned}
$$


This implies

$$
\begin{aligned}
& \int_{D_{f}^{-}(s)}(\alpha-1) f^{\alpha-2}\|\nabla f\|^{2} d V \leq \int_{D_{f}^{-}(2 s)}(\alpha-1) \phi^{2}(f) f^{\alpha-2}\|\nabla f\|^{2} d V \\
& \leq \quad \int_{D_{f}^{-}(2 s)}\left(\frac{\tilde{C}}{s^{2}}\|\nabla f\|^{2} f^{\alpha}+\frac{\tilde{C}}{s} \Delta f f^{\alpha}\right) d V \\
& \quad+\int_{D_{f}^{-}(2 s)}\left((\alpha-1) f^{\alpha-1} \frac{\tilde{C}}{s}\|\nabla f\|^{2}+\frac{4}{(\alpha-1)} \frac{\tilde{C}}{s^{2}}\|\nabla f\|^{2} f^{\alpha}\right) d V .
\end{aligned}
$$

Assuming $\frac{\|B\|^{2}}{\cos ^{4+\alpha} \theta}$ integrable on $M$, by Lemma $3.1\|\nabla f\|^{2} f^{\alpha}$, and so $\|\nabla f\|^{2} f^{\alpha-1}$, are integrable, and under the condition of integrability of $\frac{\sin \theta}{\cos ^{\alpha+2} \theta}\|\bar{R}\|$ we obtain the integrability of $\Delta f f^{\alpha}$. Making $s \rightarrow+\infty$, from the above inequality we have

$$
\int_{M}(\alpha-1) f^{\alpha-2}\|\nabla f\|^{2} d V=\lim _{s \rightarrow+\infty} \int_{D_{f}^{-}(s)}(\alpha-1) f^{\alpha-2}\|\nabla f\|^{2} d V=0
$$

and $f$ is constant, what is impossible unless $M$ is compact.

\section{Some calibrations}

\subsection{The volume calibration}

We consider in a Riemannain product $\bar{M}=M \times N$ of two Riemannian manifolds $\left(M, g_{1}\right)$ and $(N, h)$ the volume calibration (1.2), and $M$ a graph submanifold $F=\Gamma_{f}: M \rightarrow M \times N$ of a map $f: M \rightarrow N$. The graph metric on $M$ is the induced metric $g=g_{1}+f^{*} h$ by the graph map $\Gamma_{f}(p)=(p, f(p))$. We take $a_{i}$ a diagonalizing $g_{1}$-orthonormal basis of $f^{*} h$ with eigenvalues $\lambda_{1}^{2} \geq \lambda_{2}^{2} \geq \cdots \geq \lambda_{m}^{2} \geq 0$. Let $k$ such that $\lambda_{k}^{2}>0$ and $\lambda_{k+1}^{2}=0$, and consider the orthonormal system of $T_{f(p)} N, a_{1+m}, \ldots a_{1+k}$ defined by $d f\left(a_{i}\right)=\lambda_{i} a_{i+m}$, and extend to an orthonormal basis $a_{1+m}, \ldots, a_{n+m}$. Then for $i=1, \ldots, m$, $\alpha=1, \ldots, n$ (where $\lambda_{\alpha}=0$ for $\alpha \geq k+1$ )

$$
X_{i}=\frac{d \Gamma_{f}\left(a_{i}\right)}{\sqrt{1+\lambda_{i}^{2}}}=\frac{a_{i}+\lambda_{i} a_{i+m}}{\sqrt{1+\lambda_{i}^{2}}}, \quad X_{m+\alpha}=\frac{\lambda_{\alpha} a_{\alpha}-a_{\alpha+m}}{\sqrt{1+\lambda_{\alpha}^{2}}}
$$

define respectively an orthonormal basis of $\left(T_{(p, f(p))} \Gamma_{f}, g\right)$ and of $\left(N M_{p}, \bar{g}\right)$. The sign of $\lambda_{i}$ can be chosen such that $X_{i}$ is a direct basis of $\Gamma_{f}$. Then considering $\Phi$ as a morphism from $T_{(p, f(p))} \Gamma_{f}$ to $N M_{p}$ we have

$$
\Phi\left(X_{i}\right)=\cos \theta \lambda_{i} X_{i+m}, \quad \cos \theta=\left(\Pi_{j}\left(1+\lambda_{j}^{2}\right)\right)^{-1 / 2}
$$


and as a morphism from $T M, \Phi\left(a_{i}\right)=\cos \theta\left(\left(d f^{t} d f\left(a_{i}\right),-d f\left(a_{i}\right)\right)\right.$, where $d f^{t}$ is the adjoint map. For $B=\sum_{i j a} h_{i j}^{a} X_{a}, a=m+1, \ldots, m+n$ we have

$$
\|B\|^{2} \geq \sum_{i j k}\left(h_{i k}^{m+j}\right)^{2}=\sum_{i<j, k}\left[\left(h_{i k}^{m+j}\right)^{2}+\left(h_{j k}^{m+i}\right)^{2}\right]+\sum_{i k}\left(h_{i k}^{m+i}\right)^{2} .
$$

If $n=1, Q_{\Omega}(B) \geq\|B\|^{2}$. For $n \geq 2$ if we assume $\left|\lambda_{i} \lambda_{j}\right| \leq 1-\delta$ for $i \neq j$, where $0<\delta \leq 1$, the quadratic form $Q_{\Omega}(B)$ is also $\delta$-positive. Indeed, we have $([37])$

$$
\begin{aligned}
Q_{\Omega}(B)= & \|B\|^{2}+\sum_{i k} \lambda_{i}^{2}\left(h_{i k}^{m+i}\right)^{2}+2 \sum_{k, i<j} \lambda_{i} \lambda_{j} h_{j k}^{m+i} h_{i k}^{m+j} \\
= & \delta\|B\|^{2}+(1-\delta)\|B\|^{2}+\sum_{i k} \lambda_{i}^{2}\left(h_{i k}^{m+i}\right)^{2}+2 \sum_{k, i<j} \lambda_{i} \lambda_{j} h_{j k}^{m+i} h_{i k}^{m+j} \\
\geq & \delta\|B\|^{2}+(1-\delta)\left(\sum_{i<j, k}\left[\left(h_{i k}^{m+j}\right)^{2}+\left(h_{j k}^{m+i}\right)^{2}\right]+\sum_{i k}\left(h_{i k}^{m+i}\right)^{2}\right) \\
& -(1-\delta) \sum_{i k}\left(h_{i k}^{m+i}\right)^{2}-2(1-\delta) \sum_{i<j, k}\left|h_{i k}^{m+j}\right|\left|h_{j k}^{m+i}\right| \geq \delta\|B\|^{2}
\end{aligned}
$$

Moreover, $\cos ^{2} \theta\left(\sum_{i} \lambda_{i}^{2}\right) \leq \sin ^{2} \theta \leq(m-1) \cos ^{2} \theta\left(\sum_{i} \lambda_{i}^{2}\right)$, for $\sin ^{2} \theta=1-\cos ^{2} \theta=\cos ^{2} \theta\left(\Pi_{i}\left(1+\lambda_{i}^{2}\right)-1\right)=\cos ^{2} \theta\left(\sum_{i} \lambda_{i}^{2}+\sum_{i<j} \lambda_{i}^{2} \lambda_{j}^{2}+\ldots\right)$.

We denote by $\nabla d f$ the Hessian of $f:\left(M, g_{1}\right) \rightarrow(N, h)$. Let $g_{i j}=g\left(a_{i}, a_{j}\right)=$ $\delta_{i j}\left(1+\lambda_{i}^{2}\right)$ and consider the section $W$ of $f^{-1} T N$ and the vector field $Z_{1}$ of $M$ :

$$
W=\text { trace }_{g} \nabla d f=\sum_{i j} g^{i j} \nabla d f\left(a_{i}, a_{j}\right), \quad Z_{1}=\sum_{s t} g^{s t} h\left(W, d f\left(a_{s}\right)\right) a_{t} .
$$

Then (see [30]) $m H=\left(-Z_{1}, W-d f\left(Z_{1}\right)\right)$. Assuming $H$ is parallel with $c=\|H\|$, the vector field $Z$ we used in the proof of Theorem 1.2 can be expressed as $Z=-\frac{\cos \theta}{m} Z_{1}$. We have the relations $\left\|Z_{1}\right\|_{g_{1}} \leq m c, d i v_{g_{1}}\left(Z_{1}\right)=$ $m^{2} c^{2},\|Z\|_{g} \leq|\sin \theta| c, \operatorname{div}_{g}(Z)=-m c^{2} \cos \theta$. Integration on $D$ of $\operatorname{div}_{g_{1}}\left(Z_{1}\right)$ gives Theorem 1.1.

\subsection{The foliation calibration}

Assume $\pi: \bar{M} \rightarrow N$ is a transversally oriented $m$-foliation of an oriented Riemannian manifold $\left(\bar{M}^{m+n}, \bar{g}\right)$ onto a set $N$ with $\bar{M}=\cup_{y \in N} M_{y}$, where the leaf at $y, M_{y}=\pi^{-1}(y)$, is an oriented $m$-submanifold, and such that for each $x \in \bar{M}$, we have a split $T_{x} \bar{M}=T_{x} \bar{M}^{v} \oplus T_{x} \bar{M}^{h}$ where the vertical space $T_{x} \bar{M}^{v}=T_{x}\left(M_{\pi(x)}\right)$ is orthogonal to the horizontal space $T_{x} \bar{M}^{h}$, defining smooth oriented vector subbundles of $T \bar{M}$. If $N$ is a smooth $n$-manifold, $\pi$ is a fibration if $\forall x, \operatorname{Kern} d \pi(x)=T_{x} \bar{M}^{v}$ and $d \pi(x): T_{x} \bar{M}^{h} \rightarrow T_{\pi(x)} N$ is an isomorphism, and it is Riemannian if $N$ has a metric $h$ such that $d \pi(x)$ is an isometry for any $x$. We define for $X_{i}$ vector fields of $\bar{M}$

$$
\Omega\left(X_{1}, \ldots, X_{m}\right)=\operatorname{Vol}_{\pi(x)}\left(X_{1}^{v}, \ldots, X_{m}^{v}\right)
$$


where $\operatorname{Vol}_{\pi(x)}$ is the volume element of the leaf at $p=\pi(x)$. Let $e_{a}, a=$ $1, \ldots, m+n$, be a local orthonormal frame of $\bar{M}$ with $e_{i}, i=1, \ldots, m$, vertical and $e_{\alpha}, \alpha=m+1, \ldots, m+n$, horizontal. We denote by $B_{x}^{v}\left(e_{j}, e_{i}\right)=\left(\bar{\nabla}_{e_{j}} e_{i}\right)^{h}$ the second fundamental form of the leaf $M_{\pi(x)}$ and $H_{x}^{v}$ the mean curvature at $x$. We assume $e_{i}$ is a direct basis of the leaf. Now we have

$$
\bar{\nabla}_{e_{a}} \Omega\left(e_{\alpha}, e_{1}, \ldots, \hat{e}_{i}, \ldots, e_{m}\right)=-\Omega\left(\bar{\nabla} e_{a} e_{\alpha}, e_{1}, \ldots, \hat{e}_{i}, \ldots, e_{m}\right)=(-1)^{i} \bar{g}\left(\bar{\nabla} e_{a} e_{\alpha}, e_{i}\right) .
$$

From this equality and other similar ones, follows the following lemma:

Lemma 5.1. All components of $\bar{\nabla} \Omega$ and of $d \Omega$ vanish except for the following where $i, j \leq m, \alpha, \beta \geq m+1$

$$
\begin{aligned}
& \bar{\nabla}_{e_{j}} \Omega\left(e_{\alpha}, e_{1}, \ldots, \hat{e}_{i}, \ldots, e_{m}\right)=(-1)^{i+1} \bar{g}\left(B^{v}\left(e_{j}, e_{i}\right), e_{\alpha}\right) \\
& \bar{\nabla}_{e_{\beta}} \Omega\left(e_{\alpha}, e_{1}, \ldots, \hat{e}_{i}, \ldots, e_{m}\right)=(-1)^{i} \bar{g}\left(\bar{\nabla}_{e_{\beta}} e_{\alpha}, e_{i}\right) \\
& d \Omega\left(e_{\alpha}, e_{1}, \ldots, e_{m}\right)=-m \bar{g}\left(H^{v}, e_{\alpha}\right) \\
& d \Omega\left(e_{\alpha}, e_{\beta}, e_{1}, \ldots, \hat{e}_{i}, \ldots, e_{m}\right)=(-1)^{i} \bar{g}\left(\left[e_{\alpha}, e_{\beta}\right], e_{i}\right)
\end{aligned}
$$

So, $d \Omega=0$ iff $H^{v}=0$ and $\left[T \bar{M}^{h}, T \bar{M}^{h}\right] \subset T \bar{M}^{h}$, and $\bar{\nabla} \Omega=0$ iff $B^{v}=0$ and $\left(\bar{\nabla}_{T \bar{M}^{h}} T \bar{M}^{h}\right)^{v}=0$. Therefore, we can conclude:

Proposition 5.1. $\Omega$ is a calibration in $\bar{M}$, that is $d \Omega=0$, if and only if the leaves are minimal and the horizontal subspace defines an integrable distribution $T \bar{M}^{h}$ of rank $n$. In this case $\Omega$ calibrates the leaves and consequently they are stable minimal submanifolds. It defines a parallel calibration if and only if the leaves and the integral submanifolds of $\bar{M}^{h}$ are all totally geodesic.

Corollary 5.1. If $n=1$ and $\pi$ defines a foliation of $\bar{M}$ by minimal hypersurfaces, then $\Omega$ defines a closed calibration and the leaves are stable. $\Omega$ is a parallel calibration if and only if the leaves are totally geodesic and the unit normal $\bar{X}$ to the leaves satisfies $\bar{\nabla}_{\bar{X}} \bar{X}=0$. This is the case when for some function $f: M \rightarrow \mathbb{R}, \bar{Y}=f \bar{X}$ defines a nondegenerated Killing vector field with $f$ constant along each leaf.

Proof. We only have to prove the last statement. From integrability of the leaves, one easily sees that

$$
0=\bar{g}\left(\left[e_{i}, e_{j}\right], \bar{Y}\right)=2 \bar{g}\left(e_{i}, \bar{\nabla}_{e_{j}} \bar{Y}\right)=-2 \bar{g}\left(B^{v}\left(e_{i}, e_{j}\right), \bar{Y}\right) .
$$

Moreover, $f \bar{g}\left(\bar{\nabla}_{\bar{X}} \bar{X}, e_{i}\right)=-\bar{g}\left(\bar{\nabla}_{e_{i}} \bar{Y}, \bar{X}\right)=-d f\left(e_{i}\right)$.

If $n=1$ the mean curvature and the second fundamental form of the leaves have been studied by Barbosa, Kenmotsu, Oshikiri, Bessa and Montenegro in $[5,4]$, for the general case $\Omega$ closed. The above Corollary 5.1, 
using the fact $\Omega$ is a closed calibration, gives an elementary proof of the stability of the leaves (see introduction) proved in [4] using more classical stability arguments involving eigenvalue problems and maximum principles.

Corollary 5.2. Let $(N, h)$ be a Riemannian manifold and $G$ a Lie group and $N^{\prime} \subset G$ a subset that acts transitively and freely on $N$ as a group of isometries, and $f: M \rightarrow N$ a smooth map defining a minimal graph $\Gamma_{f}$ of $M \times N$. If the orbit $\left(I d_{M} \times N^{\prime}\right) N M$ of the normal bundle of $\Gamma_{f}$ defines an integrable distribution of $M \times N$, then $\Gamma_{f}$ is stable. Furthermore, if $G=N=N^{\prime}$, and $\mathfrak{g}=T_{e} G$, e the identity element, then the later condition is equivalent to $\psi=-d f^{t}: \mathfrak{g} \rightarrow C^{\infty}(T M)$ is a Lie algebra homomorphism. In particular all minimal graphs in $M \times \mathbb{R}$ are stable.

Proof. This argument is used in [4] for $N=G=\mathbb{R}$ to construct a foliation in $M \times N$. Let $a f(p)=L_{a}(f(p))$, where $a \in N^{\prime} \subset G$ acts on the left of $N$, and $a$ on $T_{y} N$ acts as $\left(L_{a}\right)_{* y}$. Since the action is free and transitive then $M \times N=\cup_{a \in G} \Gamma_{a f}$ is a foliation, and since each $a$ is an isometry, $\Gamma_{a f}$ is a minimal submanifold. Now, the normal bundle of $\Gamma_{a f}$ is just given by $\left(I d \times\left(L_{a}\right)_{*}\right)(N M)=\left\{\left(-d f_{p}^{t}(Y),\left(L_{a}\right)_{* f(p)} Y\right): Y \in T_{f(p)} N\right\}$, and we get the integrability condition on the orbit. If $G=N=N^{\prime}$, this means $\left\{\left(-d f_{p}^{t}\left(\tilde{Y}_{f(p)}\right), \tilde{Y}_{y}\right), \tilde{Y} \in \mathfrak{g}, y \in G, p \in M\right\}$ is an integrable distribution, where $\tilde{Y}_{y}=\left(L_{y}\right)_{* e}\left(\tilde{Y}_{e}\right)$. We easily see this is equivalent to $[\psi(\tilde{X}), \psi(\tilde{Y})]_{M}=$ $\psi([\tilde{X}, \tilde{Y}])$, where $\psi(\tilde{X})$ defines a vector field on $M$, that at $p \in M$ values $-d f_{p}^{t}\left(\tilde{X}_{f(p)}\right)$.

Remark. In the previous corollary, if $m=n, M=\mathbb{R}^{n}$ and $f=\nabla \phi$ where $\phi: \mathbb{R}^{n} \rightarrow \mathbb{R}$ is a smooth function, defining a minimal Lagrangian graph in $\mathbb{R}^{n} \times \mathbb{R}^{n}$, then $\psi\left(\partial_{i}\right)=$ Hess $\phi^{\sharp}\left(\partial_{i}\right)$. The stability condition means $D^{3} \phi\left(\right.$ Hess $\left.\phi^{\sharp}\left(\partial_{i}\right), \partial_{j}, \partial_{k}\right)=D^{3} \phi\left(\right.$ Hess $\left.\phi^{\sharp}\left(\partial_{j}\right), \partial_{i}, \partial_{k}\right)$. This seems to be quite different from the condition of special Lagrangian graphs. Minimal Lagrangian graphs are calibrated by the special Lagrangian calibration ([17]), and may not satisfy the above condition, that is related to a different calibration.

Corollary 5.3. Any minimal submanifold $M^{m}$ of $\mathbb{R}^{m+n}$, with $n=1$, is locally stable and locally a calibrated submanifold. The same also holds for $n \geq 2$ if for a local representation as a graph of a map $f: \mathbb{R}^{m} \rightarrow \mathbb{R}^{n}$, $\psi=-d f^{t}: \mathbb{R}^{n} \rightarrow C^{\infty}\left(T \mathbb{R}^{m}\right)$ is a Lie algebra homomorphism.

Proof. Let $p \in M$ and $D$ a compact neighbourhood of $p$ where it is defined $\nu_{\alpha}$, an orthonormal frame of $N M$ defined on $D$. We identify $L=T_{p} M$ with $\mathbb{R}^{m}$. Now $d \pi_{L}(p)$ is the identity map of $T_{p} M$ where $\pi_{L}(q)=q-$ $\sum_{\alpha} \bar{g}\left(q, \nu_{\alpha}(p)\right) \nu_{\alpha}(p)$ and we may assume $\pi_{L}$ is a diffeomorphism. Thus, $D$ is the graph of a map $f: \mathbb{R}^{m} \rightarrow \mathbb{R}^{n}$. 
Next we generalize the main result of [5] (Proposition 2.14) for any codimension. We assume $T \bar{M}^{h}$ is an integrable distribution and consider the maximal horizontal integrable $n$-submanifold $\Sigma$ passing at a given pont $x \in \bar{M}$, with second fundamental form $B_{x}^{h}\left(e_{\alpha}, e_{\beta}\right)=\left(\bar{\nabla}_{e_{\alpha}} e_{\beta}\right)^{v}$, and mean curvature $H_{x}^{h}=\frac{1}{n} \sum_{\alpha} B_{x}^{h}\left(e_{\alpha}, e_{\alpha}\right)$. Let $s^{v}$ be the scalar curvatures of the leaves and $s^{h}$ the ones of the horizontal integrable submanifolds, and $\overline{\text { Ricci }}$ and $\bar{s}$ the Ricci tensor and scalar curvature of $\bar{M}$, respectively. $H^{h}$ defines a vertical vector field of $\bar{M}$, and consider its divergence in a fiber and in $\bar{M}$ respectively:

$$
\operatorname{div}\left(H^{h}\right)=\sum_{i} \bar{g}\left(\bar{\nabla}_{e_{i}} H^{h}, e_{i}\right), \quad \overline{\operatorname{div}}\left(H^{h}\right)=\sum_{a} \bar{g}\left(\bar{\nabla}_{e_{a}} H^{h}, e_{a}\right)
$$

Similarly for the horizontal vector field $H^{v}$ we may take its divergence along an horizontal integrable submanifold $\Sigma, \operatorname{div}_{\Sigma}\left(H^{v}\right)=\sum_{\alpha} \bar{g}\left(\bar{\nabla}_{e_{\alpha}} H^{v}, e_{\alpha}\right)$.

Lemma 5.2. Assuming $T \bar{M}^{h}$ is an integrable distribution

$$
\begin{aligned}
\overline{\operatorname{div}}\left(H^{h}\right) & =\operatorname{div}\left(H^{h}\right)-n\left\|H^{h}\right\|^{2} \\
\overline{\operatorname{div}}\left(H^{v}\right) & =\operatorname{div}_{\Sigma}\left(H^{v}\right)-m\left\|H^{v}\right\|^{2} \\
n \operatorname{div}\left(H^{h}\right)+m \operatorname{div}_{\Sigma}\left(H^{v}\right) & =\sum_{i} \overline{\operatorname{Ricci}}\left(e_{i}, e_{i}\right)-s^{v}+m^{2}\left\|H^{v}\right\|^{2}+\left\|B^{h}\right\|^{2} \\
& =\sum_{\alpha} \overline{\operatorname{Ricci}}\left(e_{\alpha}, e_{\alpha}\right)-s^{h}+n^{2}\left\|H^{h}\right\|^{2}+\left\|B^{v}\right\|^{2} . \\
2 n \operatorname{div}\left(H^{h}\right)+2 m \operatorname{div}_{\Sigma}\left(H^{v}\right) & = \\
=\bar{s}-s^{v}- & s^{h}+m^{2}\left\|H^{v}\right\|^{2}+n^{2}\left\|H^{h}\right\|^{2}+\left\|B^{h}\right\|^{2}+\left\|B^{v}\right\|^{2} .
\end{aligned}
$$

Proof. We may assume at a point $x,\left(\bar{\nabla}_{e_{a}} e_{i}(x)\right)^{v}=\left(\bar{\nabla}_{e_{a}} e_{\alpha}(x)\right)^{h}=0$, $\forall a, i, \alpha$. The first two equalities are obtained by an elementary computation. At $x$

$$
\begin{aligned}
n \operatorname{div}\left(H^{h}\right) & \left.=\sum_{i, \alpha} \bar{g}\left(\bar{\nabla}_{e_{i}}\left(\bar{\nabla}_{e_{\alpha}} e_{\alpha}\right)^{v}\right), e_{i}\right)=\sum_{i, \alpha} d\left(\bar{g}\left(\left(\bar{\nabla}_{e_{\alpha}} e_{\alpha}\right)^{v}, e_{i}\right)\right)\left(e_{i}\right) \\
& =\sum_{i, \alpha} d\left(\bar{g}\left(\bar{\nabla}_{e_{\alpha}} e_{\alpha}, e_{i}\right)\right)\left(e_{i}\right)=\sum_{i, \alpha} \bar{g}\left(\bar{\nabla}_{e_{i}} \bar{\nabla}_{e_{\alpha}} e_{\alpha}, e_{i}\right) \\
& =\bar{g}\left(\bar{R}\left(e_{\alpha}, e_{i}\right) e_{\alpha}+\bar{\nabla}_{e_{\alpha}} \bar{\nabla}_{e_{i}} e_{\alpha}+\bar{\nabla}_{\left[e_{i}, e_{\alpha}\right]} e_{\alpha}, e_{i}\right) .
\end{aligned}
$$

and

$$
\begin{aligned}
& \sum_{i} \bar{g}\left(\bar{\nabla}_{e_{\alpha}} \bar{\nabla}_{e_{i}} e_{\alpha}, e_{i}\right)=\sum_{i}-\bar{\nabla}_{e_{\alpha}}\left(\bar{g}\left(e_{\alpha}, \overline{\nabla_{e_{i}}} e_{i}\right)\right)=-\bar{g}\left(e_{\alpha}, m \bar{\nabla}_{e_{\alpha}} H^{v}\right) \\
& \bar{g}\left(\bar{\nabla}_{\nabla e_{i} e_{\alpha}} e_{\alpha}, e_{i}\right)=\sum_{j} \bar{g}\left(\bar{\nabla}_{e_{j}} e_{\alpha}, e_{i}\right) \bar{g}\left(\bar{\nabla}_{e_{i}} e_{\alpha}, e_{j}\right) \\
& \bar{g}\left(\bar{\nabla}_{\nabla e_{\alpha} e_{i}} e_{\alpha}, e_{i}\right)=\sum_{\beta} \bar{g}\left(\bar{\nabla}_{e_{\beta} e_{\alpha}}, e_{i}\right) \bar{g}\left(\bar{\nabla}_{e_{\alpha}} e_{i}, e_{\beta}\right)
\end{aligned}
$$

leading to $\sum_{i, \alpha} \bar{g}\left(\bar{\nabla}_{\left[e_{i}, e_{\alpha}\right]} e_{\alpha}, e_{i}\right)=\left\|B^{h}\right\|^{2}+\left\|B^{v}\right\|^{2}$, and consequently,

$$
n \operatorname{div}\left(H^{h}\right)=\sum_{\alpha, i} \bar{R}\left(e_{\alpha}, e_{i}, e_{\alpha}, e_{i}\right)+\left\|B^{h}\right\|^{2}+\left\|B^{v}\right\|^{2}-m \operatorname{div}_{\Sigma}\left(H^{v}\right) .
$$


Now $\sum_{i \alpha} \bar{R}\left(e_{\alpha}, e_{i}, e_{\alpha}, e_{i}\right)=\sum_{i} \overline{\operatorname{Ricci}}\left(e_{i}, e_{i}\right)-\sum_{i j} \bar{R}\left(e_{j}, e_{i}, e_{j}, e_{i}\right)$ and using Gauss equation with respect to a leaf, we obtain the first expression for $n \operatorname{div}\left(H^{h}\right)$. Writing $\sum_{i \alpha} \bar{R}\left(e_{\alpha}, e_{i}, e_{\alpha}, e_{i}\right)=\sum_{\alpha} \overline{\operatorname{Ricci}}\left(e_{\alpha}, e_{\alpha}\right)-\sum_{\alpha \beta} \bar{R}\left(e_{\alpha}, e_{\beta}\right.$, $\left.e_{\alpha}, e_{\beta}\right)$ and applying Gauss equations with respect to $\Sigma$ we get the second expression for $n \operatorname{div}\left(H^{h}\right)$. Summing the previous two expressions we obtain the last one.

Proposition 5.2. Assume $\bar{M}$ is closed, $T \bar{M}^{h}$ is an integrable distribution and $H^{v}$ is a divergence free vector field along each horizontal integral submanifold. Then $H^{v}=0$, i.e. the leaves are minimal and $d \Omega=0$. Furthermore:

(1) If $H^{h}$ is also a divergence free vector field along each leaf, the horizontal integral submanifolds are minimal as well.

(2) If $\overline{\text { Ricci }} \geq 0$ and $s^{v} \leq 0$ for all the leaves, the horizontal integrable submanifolds are totally geodesic and $\overline{\text { Ricci }}$ vanish in the direction of all leaves and $s^{v}=0$.

(3) If $\overline{\text { Ricci }} \geq 0$ and $s^{h} \leq 0$ for all horizontal integral submanifolds $\Sigma$, then they are minimal and the leaves of $\pi$ are totally geodesic and $\overline{\text { Ricci }}$ vanish in the direction of all horizontal vector fields and $s^{h}=0$.

(4) If $\bar{s} \geq 0$ and $s^{v}+s^{h} \leq 0$, all the leaves and the horizontal integrable submanifolds are totally geodesic, and $\bar{s}=s^{v}+s^{h}=0$.

Consequently, if $\bar{s} \geq 0(\overline{\text { Ricci }} \geq 0$ resp.) and $\bar{s}>0(\overline{\text { Ricci }}>0$ resp. $)$ at some point $x$, then either the leaf $M_{\pi(x)}$ or (and resp.) the horizontal integral submanifold at some $y \in \pi^{-1}(x)$ must have positive scalar curvature somewhere. In particular, in the later case, $n \geq 2$.

Proof. If $\operatorname{div}_{\Sigma}\left(H^{v}\right)=0$ for each $\Sigma$, integration of $\overline{\operatorname{div}}\left(H^{v}\right)$ on $\bar{M}$ of the second formula of previous lemma gives $H^{v}=0$. Similarly for (1), using the first formula. To prove (2) (3) and (4) we integrate the last three formulas of Lemma 5.2, with $H^{v}=0$, along each leaf $M_{\pi(x)}$, that is compact.

If $n=1$ and $H^{v}$ is constant, with the same constant for all fibers, then $H^{v}$ is a divergence free vector along each horizontal integral submanifold, giving the case in [5].

\subsection{The Kähler calibration}

On a Kähler manifold $(\bar{M}, J, \bar{g})$ with Kähler form $w(X, Y)=g(J X, Y)$ it is defined the Kähler calibration $\Omega=\frac{w^{k}}{k !}$, that calibrates the complex submanifolds of complex dimension $k$. If $\theta_{1}, \ldots, \theta_{k}$ are the Kähler angles of $M$, $\cos \theta_{1} \geq \cdots \geq \cos \theta_{k} \geq 0$ and $e_{a}=X_{i}, Y_{i}$ a diagonalizing o.n basis of $F^{*} w$, 
that is $F^{*} w\left(X_{i}, X_{j}\right)=F^{*} w\left(Y_{i}, Y_{j}\right)=0, F^{*} w\left(X_{i}, Y_{j}\right)=\cos \theta_{i} \delta_{i j}$, then

$$
\cos \theta=\epsilon \cos \theta_{1} \cdots \cos \theta_{k}, \quad \epsilon= \pm 1
$$

and $\Phi\left(X_{i}\right)=-\epsilon \cos \theta\left(J\left(\frac{Y_{i}}{\cos \theta_{i}}\right)\right)^{\perp}, \quad \Phi\left(Y_{i}\right)=\epsilon \cos \theta\left(J\left(\frac{X_{i}}{\cos \theta_{i}}\right)\right)^{\perp}$. A submanifold $M$ is said to have equal Kähler angles, if $\epsilon=1$ and $\theta_{i}=\vartheta \forall i$ (see [34]). It is a complex (resp. Lagrangian) submanifold iff $\cos \vartheta=1($ resp. $\cos \vartheta=0)$. We assume $M$ and $\bar{M}$ are of real and complex dimension 4 respectively and $M$ has equal Kähler angles. We recall that $\left(F^{*} w\right)^{\sharp}: T M \rightarrow T M$ and $\Phi: T M \rightarrow N M$ (with respect to the Kähler calibration) are conformal morphisms with coefficient of conformality $\cos ^{2} \vartheta$ and $\sin ^{2} \vartheta \cos ^{2} \vartheta$, respectively. Note that $\Phi=-\Phi^{\prime} \circ\left(F^{*} w\right)^{\sharp}$ with $\Phi^{\prime}(X)=(J X)^{\perp}$, given in $[34,33]$. We have $\left\|\Phi^{\prime}(X)\right\|^{2}=\sin ^{2} \vartheta\|X\|^{2}$. We can write $\left(F^{*} w\right)^{\sharp}=\cos \vartheta J_{w}$ where $J_{w}$ is the almost complex structure of $M$, defined where $\cos \vartheta \neq 0$ by $J_{w}\left(X_{i}\right)=Y_{i}$. Similarly we get a polar decomposition for $w^{\perp}=\cos \vartheta J^{\perp}$, the restriction of $w$ to the normal bundle. The orthonormal frame of the normal bundle $U_{i}=\Phi^{\prime}\left(\frac{Y_{i}}{\sin \vartheta}\right), V_{i}=J^{\perp} U_{i}=\Phi^{\prime}\left(\frac{X_{i}}{\sin \vartheta}\right)$ diagonalizes $w^{\perp}$. We have

$$
\begin{aligned}
\Omega\left(X_{i}, Y_{j}, U_{k}, V_{s}\right) & =\left(\cos ^{2} \vartheta+\sin ^{2} \vartheta \delta_{i k}\right) \delta_{i j} \delta_{k s} \\
\Omega\left(X_{1}, X_{2}, V_{1}, V_{2}\right) & =\Omega\left(Y_{1}, Y_{2}, U_{1}, U_{2}\right)=-\sin ^{2} \vartheta
\end{aligned}
$$

and all the other components of $\Omega$ in this basis vanish. Then it follows the condition $\tilde{Q}_{\Omega}(B) \geq \delta\|B\|^{2}$ is very restrictive. Consider the complex and anticomplex parts of $B$ with respect the almost complex structures $J_{w}$ of $T M$ and $J^{\perp}$ of $N M$ :

$$
\begin{aligned}
& B^{c}(X, Y)=\frac{1}{2}\left(B(X, Y)-J^{\perp} B\left(J_{w} X, Y\right)\right), \\
& B^{a}(X, Y)=\frac{1}{2}\left(B(X, Y)+J^{\perp} B\left(J_{w} X, Y\right)\right) .
\end{aligned}
$$

Then $\tilde{Q}(B)=\|B\|^{2}+(\cos \theta)^{-1}\left(\left\|B^{a}\right\|^{2}-\left\|B^{c}\right\|^{2}\right)+\rho$, where

$$
|\rho| \leq 4 \frac{\sin ^{2} \vartheta}{\cos \theta} \sum_{a<b, c}\left\|B\left(e_{a}, e_{c}\right)\right\|\left\|B\left(e_{b}, e_{c}\right)\right\| \leq 12 \frac{\sin ^{2} \vartheta}{\cos \theta}\|B\|^{2} .
$$

Using this upper bound, for $\cos \theta \in\left(\frac{11}{13}, \frac{11}{12}\right]$, if

$$
\left\|B^{a}\right\|^{2} \geq \frac{(13-\cos \theta(13-\delta))}{(-11+\cos \theta(13-\delta))}\left\|B^{c}\right\|^{2}, \quad \text { with } \quad 0 \leq \delta<\frac{13 \cos \theta-11}{\cos \theta} \leq 1
$$

we have $\tilde{Q}(B) \geq \delta\|B\|^{2}$. Note that if $M$ is a complex submanifold, $J_{w}=$ $J^{\perp}=J, \sin \vartheta=0, B$ is a complex bilinear form, and $\tilde{Q}(B)=0$. So, calibrated submanifolds may not be totally geodesic. Theorem 1.5(A) gives (3) of next proposition 
Proposition 5.3. Let $F: M^{2 k} \rightarrow \bar{M}^{2 k}$ be a $2 k$-submanifold immersed with parallel mean curvature $H$ and with equal Kähler angles into a Kähler manifold of complex dimension $2 k$ and scalar curvature $\bar{s}$.

(1) [34] Assume $H=0$ and $\bar{M}$ is Einstein. If $k=2$ and $\bar{s} \neq 0$, then $F$ is either a complex or a Lagrangian submanifold. If $k \geq 3, \bar{s}<0$, and $M$ closed, then $F$ is either complex or Lagrangian. If $k \geq 3, \bar{s}=0$, and $M$ closed, then $\theta$ is constant.

(2) [31] If $k=2, \bar{s}<0, M$ closed and $\|H\|^{2} \geq-(\bar{s} / 8) \sin ^{2} \vartheta$, then $F$ is either a complex or a Lagrangian submanifold.

(3) If $k=2, \cos \theta>0,(1.7)$ holds and (5.1) is satisfied for some $\delta$ and $\cos \theta \in\left(\frac{11}{13}, \frac{11}{12}\right]$, and $M$ is closed, then $F$ is totally geodesic.

If $\bar{M}$ is a complex space form of sectional holomorphic curvature $\nu$ then for $U \in N M$,

$$
\bar{R}\left(e_{a}, e_{b}, e_{a}, U\right)=\frac{3 \nu}{4} w\left(e_{a}, e_{b}\right) \bar{g}\left(\Phi^{\prime}\left(e_{a}\right), U\right),
$$

and

$$
\sum_{a b} \bar{R}\left(e_{a}, e_{b}, e_{a}, \Phi^{\prime}\left(e_{b}\right)\right)=\frac{3 k \nu}{2} \cos ^{2} \vartheta \sin ^{2} \vartheta .
$$

Note that $\nu$ has the same sign has $\bar{s}$, but for $k=2$ and $\nu>0$ (5.1) in (3) does not hold because of (1). Hence, $\delta$-positiveness of $\tilde{Q}$ can be expected only when $\bar{s}=0$. (2) is related to a result obtained by Kenmotsu and Zhou in [23], and Hirakawa in [20] where a classification of surfaces with parallel mean curvature in a complex space forms is obtained using the Kähler angle.

\subsection{The Quaternionic calibration}

This calibration is not so well understood in the literature so we will describe in some detail. Let $(V, I, J, K, g)$ be an hyper-Hermitean vector space of dimension $4 n$, where $I, J$ are two anti-commuting $g$-orthogonal structures. For each $x=(a, b, c) \in \mathbb{S}^{2}$ it is defined a $g$-orthogonal structure $J_{x}=$ $a I+b J+c K$ and its Kähler form $w_{x}(X, Y)=g\left(J_{x} X, Y\right)$. Then $V$ is a right-quaternionic vector space with $X \zeta=\zeta_{0} X-J_{x} X=: J_{\bar{\zeta}} X$ where $\zeta=$ $\left(\zeta_{0}, x\right) \in \mathbb{H}$ and $J_{x}$ is extended linearly for $x \in \mathbb{R}^{3}$. The right-quaternionic linear group of isometries of $V$ is $S p(n)=S p(V)=\left\{\xi \in O(V): \xi J_{x}=\right.$ $\left.J_{x} \xi, \forall x \in \mathbb{S}^{2}\right\} \subset S O(V)$. Let $S p(1)=\{\zeta \in \mathbb{H}:|\zeta|=1\}$. The inclusion $S p(V) \cdot S p(1)=S p(V) \times S p(1) / \pm(I d, 1) \subset S O(V)$ is given by $(\xi, \zeta) X=$ $\xi(X) \zeta^{-1}$. Moreover, for $P=(\xi, \zeta)$

$$
P\left(J_{x} X\right)=\xi\left(J_{x} X\right) \zeta^{-1}=-\xi(X) \zeta^{-1} \zeta x \zeta^{-1}=J_{\tau_{\zeta(x)}}\left(\xi(X) \zeta^{-1}\right)=J_{\tau_{\zeta(x)}} P(X)
$$

where $\tau: S^{3} \subset \mathbb{H} \rightarrow S O(3) \subset S O(4)$ is the double covering map $\tau_{\zeta}(v)=$ $\zeta v \bar{\zeta}$. A subspace $T$ is a complex subspace if $J_{x} T \subset T$ for some $x \in \mathbb{S}^{2}$. It is 
a quaternionic subspace if it is $J_{x}$-complex $\forall x$. The fundamental 4-form of $V$ is defined by

$$
\Omega=\frac{1}{6}\left(w_{I} \wedge w_{I}+w_{J} \wedge w_{J}+w_{K} \wedge w_{K}\right) .
$$

For each $X \in V$, let $H_{X}^{0}=\operatorname{span}\{I X, J X, K X\}, H_{X}=\mathbb{R} X \oplus H_{X}^{0}$. Each $P \in S O(V)$ acts on $\Omega$ as $P \Omega(X, Y, Z, W)=\Omega\left(P^{-1} X, P^{-1} Y, P^{-1} Z, P^{-1} W\right)$, and we have

Lemma 5.3. $H_{\Omega}:=\{P \in S O(V): P \cdot \Omega=\Omega\}=S p(V) \cdot S p(1)$.

Proof. If $P=(\zeta, \xi) \in \mathbb{S}^{2} \times S p(V)$ then $P=(\xi, \zeta)$ satisfies $P\left(H_{X}\right)=H_{P(X)}$. Note that $\forall X, Y \in V, w_{I}(I X, I Y)=w_{I}(X, Y), w_{J}(I X, I Y)=-w_{J}(X, Y)$. If $P=(\xi, q) \in S p(V) \cdot S p(1)$ one can prove directly that $P \cdot \Omega=\Omega$ (see [24]). Then $S p(V) \cdot S p(1) \subset H_{\Omega}$. Now if $P \in H_{\Omega}$, from the above considerations $P\left(H_{X}\right)=H_{P(X)}$. Thus, $\forall x \in \mathbb{R}^{3} P\left(J_{x} X\right)=J_{A(X, x)} P(X)$, with $A(X, \cdot) \in S O(3)$ necessarily in case $\|X\|=1$. We extend $A(X, \lambda)=\lambda$, for $\lambda \in \mathbb{R} \subset \mathbb{H}$. Since $\forall \lambda \in \mathbb{R}, P\left(J_{x} \lambda X\right)=\lambda P\left(J_{x} X\right)$ we get $A(\lambda X, x)=$ $A(X, x)$. Now we assume $\|X\|=1$. From $P\left(J_{x y} X\right)=P\left(J_{x}\left(J_{y} X\right)\right)$ we have $A(X, x y)=A\left(J_{y} X, x\right) A(X, y)$. Let $x=\mu x^{\prime}+\lambda y$, where $x^{\prime} \perp y$ is a unit of $\mathbb{R}^{3}$. Then $A\left(X, x^{\prime} \times y\right)=A\left(X, x^{\prime}\right) \times A(X, y)$, and so $A\left(J_{y} X, x\right)$ $A(X, y)=A(X, x y)=\mu A\left(X, x^{\prime} \times y\right)-\lambda I d=\mu A\left(X, x^{\prime}\right) \times A(X, y)-\lambda I d$, implying $A\left(J_{y} X, x\right)=\mu A\left(X, x^{\prime}\right)+\lambda A(X, y)=A\left(X, \mu x^{\prime}+\lambda y\right)=A(X, x)$. Finally let $X, Y$ units with $H_{X} \oplus H_{Y}$ and $Z=\frac{X+Y}{\|X+Y\|}$. Then $H_{P(X)} \oplus H_{P(Y)}$. From $P\left(J_{x}(X+Y)\right)=J_{A(Z, x)} P(X+Y)$ we get $J_{A(X, x)} P(X)+J_{A(Y, x)} P(Y)=$ $J_{A(Z, x)} P(X)+J_{A(Z, x)} P(Y)$, and so $A(X, x)=A(Z, x)=A(Y, x)$. Then $A(X, x)=A(x) \forall X$, that is $A$ does not depend on $X$. We have proved that $P\left(J_{x} X\right)=J_{\tau_{\zeta}(x)} P(X)$, where $A=\tau_{\zeta}$ for some $\zeta \in S p(1)$ (unique up to a sign). Define $\xi: V \rightarrow V$ by $\xi(X)=P(X) \zeta=J_{\bar{\zeta}} P(X)$. Then $P=(\xi, \zeta)$ and $\xi \in S p(V)$.

The fundamental 4-form induces a symmetric endomorphism $\Omega^{\Delta}: \wedge^{2} V \rightarrow$ $\wedge^{2} V$, defined by $\left\langle\Omega^{\Delta}(X \wedge Y), Z \wedge W\right\rangle=\Omega(X, Y, Z, W)$. For each oriented orthonormal system $B=\left\{X_{1}, X_{2}, X_{3}, X_{4}\right\}$ of $V$, we define the bivectors $\Lambda_{r}^{ \pm}=\Lambda_{r}^{ \pm}(B)$, by

$$
\begin{aligned}
& \Lambda_{1}^{ \pm}=\frac{1}{\sqrt{2}}\left(X_{1} \wedge X_{2} \pm X_{3} \wedge X_{4}\right), \\
& \Lambda_{2}^{ \pm}=\frac{1}{\sqrt{2}}\left(X_{1} \wedge X_{3} \mp X_{2} \wedge X_{4}\right), \\
& \Lambda_{3}^{ \pm}=\frac{1}{\sqrt{2}}\left(X_{1} \wedge X_{4} \pm X_{2} \wedge X_{3}\right) .
\end{aligned}
$$

If $X$ is a unit, $\Lambda_{r}^{ \pm}(X)$ is defined as above w.r.t. $X_{1}=X, X_{2}=I X, X_{3}=$ $J X, X_{4}=K X$. Note that $\Lambda_{r}^{ \pm}(X)=\Lambda_{r}^{ \pm}\left(J_{x} X\right)$ for any $x \in \mathbb{S}^{2}$. Set for 
any $X, Y$ and $i=0,1,2,3, r=1,2,3$, and $\epsilon_{0}^{r}=\epsilon_{1}^{3}=\epsilon_{2}^{1}=\epsilon_{3}^{2}=+1$, $\epsilon_{1}^{1}=\epsilon_{1}^{2}=\epsilon_{2}^{2}=\epsilon_{2}^{3}=\epsilon_{3}^{1}=\epsilon_{3}^{3}=-1$,

$$
\Theta_{i}(X, Y)=\frac{1}{2}\left(X \wedge Y+\epsilon_{i}^{1} I X \wedge I Y+\epsilon_{i}^{2} J X \wedge J Y+\epsilon_{i}^{3} K X \wedge K Y\right) .
$$

satisfying $\Theta_{s^{\prime}}\left(J_{x} X, J_{x} Y\right) \in \operatorname{span}\left\{\Theta_{s}(X, Y), s=0, \ldots 3\right\}$ and for $J_{x}=I, J, K$, $\Theta_{s^{\prime}}\left(X, J_{x} X\right)$ either is zero or gives $\Lambda_{s}^{ \pm}(X)$ for some $s$. If $H_{X}, H_{Y}, H_{Z}$ are orthogonal quaternionic lines, and $X, Y$ are units we have for any $x, y, z \in \mathbb{S}^{2}$

$$
\begin{aligned}
& \Omega\left(X, J_{x} X, J_{y} X, J_{z} X\right)=\langle x, y \times z\rangle \\
& \Omega\left(X, J_{x} X, Y, J_{y} Y\right)=\frac{1}{3}\langle x, y\rangle \\
& \Omega\left(X, J_{x} X, J_{y} X, Y\right)=\Omega\left(X, J_{x} X, Y, Z\right)=0 .
\end{aligned}
$$

We take an orthonormal basis $X_{i}$ of $V$ the form $\left\{e_{\alpha}, I e_{\alpha}, J e_{\alpha}, K e_{\alpha}\right\}$, $\alpha=1, \ldots, n$. We have $\Omega^{\Delta}(\xi)=\sum_{i<j} \Omega\left(\xi, X_{i} \wedge X_{j}\right) X_{i} \wedge X_{j}$. An orthonormal basis of eigenvectors of $\Omega^{\Delta}$ is given by the $2 n(4 n-1)$ vectors, where $\alpha, \beta=$ $1, \ldots, n, r=1,2,3$,

$$
\begin{aligned}
& (1 / \sqrt{n}) \sum_{\alpha} \Lambda_{r}^{+}\left(e_{\alpha}\right), \quad \Lambda_{r}^{-}\left(e_{\alpha}\right), \quad 1 / \sqrt{2}\left(\Lambda_{r}^{+}\left(e_{n}\right)-\Lambda_{r}^{+}\left(e_{\alpha}\right)\right) \quad \alpha<n \\
& \Theta_{s}\left(e_{\alpha}, I e_{\beta}\right), \quad \Theta_{s}\left(e_{\alpha}, J e_{\beta}\right), \quad \Theta_{s}\left(e_{\alpha}, K e_{\beta}\right) \quad \alpha<\beta, \quad s=0,1,2,3
\end{aligned}
$$

The corresponding eigenvalues, that range $\left\{\frac{2 n+1}{3}, \pm 1, \pm 1 / 3\right\}$, are given as follows where $i=1,2,3, L=i d, I, J, K$

$$
\begin{array}{ll}
\Omega^{\Delta}\left(\Lambda_{r}^{+}\left(e_{\alpha}\right)\right)=\Lambda_{r}^{+}\left(e_{\alpha}\right)+\sum_{\beta \neq \alpha} \frac{2}{3} \Lambda_{r}^{+}\left(e_{\beta}\right) & \Omega^{\Delta}\left(\sum_{\alpha} \Lambda_{r}^{+}\left(e_{\alpha}\right)\right)=\frac{2 n+1}{3}\left(\sum_{\alpha} \Lambda_{r}^{+}\left(e_{\alpha}\right)\right) \\
\Omega^{\Delta}\left(\Lambda_{r}^{+}\left(e_{\alpha}\right)-\Lambda_{r}^{+}\left(e_{\beta}\right)\right)=\frac{1}{3}\left(\Lambda_{r}^{+}\left(e_{\alpha}\right)-\Lambda_{r}^{+}\left(e_{\beta}\right)\right) & \Omega^{\Delta}\left(\Lambda_{r}^{-}\left(e_{\alpha}\right)\right)=-\Lambda_{r}^{-}\left(e_{\alpha}\right) \\
\Omega^{\Delta}\left(\Theta_{0}\left(e_{\alpha}, L e_{\beta}\right)\right)=\Theta_{0}\left(e_{\alpha}, L e_{\beta}\right) & \Omega^{\Delta}\left(\Theta_{i}\left(e_{\alpha}, L e_{\beta}\right)\right)=-\frac{1}{3} \Theta_{i}\left(e_{\alpha}, L e_{\beta}\right)
\end{array}
$$

Given a $k$-dimensional $T$ subspace of $V$ we consider the restriction $\Omega_{T}^{\Delta}$ : $\wedge^{2} T \rightarrow \wedge^{2} T$, symmetric endomorphism with eigenvalues $\alpha_{1}, \ldots, \alpha_{\frac{k(k-1)}{2}}$ that we call the nonnormalized quaternionic angles of $T$.

From now on we restrict our attention when $T$ is an oriented four dimensional subspace with direct orthonormal basis $\left(X_{1}, X_{2}, X_{3}, X_{4}\right)$ and $V$ eight dimensional.

Proposition 5.4. The fundamental form $\Omega$ defines a calibration that calibrates the quaternionic 4 dimensional subspaces. The quaternionic angle of an oriented 4-dimensional subspace $T^{4}$ is defined by the number $\cos \theta=$ $\Omega\left(X_{1}, X_{2}, X_{3}, X_{4}\right) \in[-1,1] . T$ and $T^{\perp}$ have the same quaternionic angle and there are only two eigenvalues $\alpha_{i}= \pm \cos \theta$ each with multiplicity three.

Proof. To see that $\Omega$ is a calibration, we set $\phi(x)=\left\langle\left(w_{x}\right)_{\mid T} \wedge\left(w_{x}\right)_{\mid T}, V o l_{T}\right\rangle$, where $\left(w_{x}\right)_{T}$ is the restriction of $w_{x}$ to $T \times T$. If $\cos \theta_{1}^{x}, \cos \theta_{2}^{x}$, with $\theta_{i}^{x} \in\left[0, \frac{\pi}{2}\right]$, 
are the $J_{x}$-Kähler angles of $T$ w.r.t. $J_{x}$, then for any o.n.b. $x, y, z$ of $\mathbb{R}^{3}$,

$$
\begin{aligned}
\cos \theta=\Omega(T) & =\frac{1}{6}(\phi(x)+\phi(y)+\phi(z)) \\
& =\frac{1}{3}\left(\epsilon_{x} \cos \theta_{1}^{x} \cos \theta_{2}^{x}+\epsilon_{y} \cos \theta_{1}^{y} \cos \theta_{2}^{y}+\epsilon_{z} \cos \theta_{1}^{z} \cos \theta_{2}^{z}\right)
\end{aligned}
$$

where $\epsilon_{u}= \pm 1$ depending if $\left(w_{u}\right)_{T}$ defines the same or the opposite orientation of $T$. From (5.5) we see that $|\phi(u)| \leq 2$ and so $|\Omega(T)| \leq 1$, and $\Omega(T)= \pm 1$ iff $\epsilon_{u}= \pm 1$ and $\cos \theta_{s}^{u}=1, s=1,2$, that is $T$ is a $J_{u}$-complex subspace, $\forall u=x, y, z$, or equivalently, $T$ is a quaternionic subspace. Since the $J_{s}$-Kähler angles of $T$ and the ones of the orthogonal complement of $T^{\perp}$ are the same, then $\Omega(T)=\Omega\left(T^{\perp}\right)$.

Proposition 5.5. If $V$ is 8-dimensional and $T$ is a 4-dimensional subspace $J_{x}$-complex for some $x$ then $\frac{1}{3} \leq \cos \theta(T) \leq 1$, with equality to $\frac{1}{3}$ if and only if $T$ is a totally complex subspace, that is $T$ is a $J_{y}$-Lagrangian subspace $\forall y \perp x$. Moreover, if two complex subspaces $T$ and $T^{\prime}$ of $V$ have the same quaternionic angle, then there exist an element $P \in S p(V) \cdot S p(1)$ such that $T^{\prime}=P(T)$.

Proof. If $T$ is a $J_{x}$-complex subspace of $V$ then $T$ is Cayley subspace of $\left(V, g, J_{y}\right)$ for all $y \in \mathbb{S}^{2}$. To see this we take an orthonormal basis of $T$ of the form $B=\left\{X_{i}\right\}=\left\{X, J_{x} X, Z, J_{x} Z\right\}$. We have $g\left(J_{y} J_{x} X, X\right)=-\langle y, x\rangle=$ $g\left(J_{y} J_{x} Z, Z\right), g\left(J_{y} J_{x} Z, X\right)=-g\left(J_{y} X, J_{x} Z\right), g\left(J_{y} Z, J_{x} X\right)=-g\left(J_{y \times x} X, Z\right)$, and $g\left(J_{y} J_{x} Z, J_{x} X\right)=g\left(J_{y} X, Z\right)$. A basis for the self-dual 2-forms on $T$ is given by $J_{r}^{B}=\Lambda_{r}^{+}(B)$. Then we see that $\left(w_{y}\right)_{\mid T}=\cos \theta^{y}(p) J_{v}^{B}$ where $v=$ $\frac{1}{t}\left(<y, x>, g\left(J_{y} X, Z\right), g\left(J_{y \times x} X, Z\right)\right) \in \mathbb{S}^{2}$ and $\cos \theta^{y}(p)=t=\left\|\left(J_{y} X\right)^{\top}\right\|$, proving that $\left(w_{y}\right)_{T}$ is self-dual, that is $*\left(w_{y}\right)_{\mid T}=\left(w_{y}\right)_{\mid T}$. This is just the same as to say the $J_{y}$-Kähler angles of $T$ are equal, that is $T$ is a Cayley subspace. Therefore $\theta_{1}^{u}=\theta_{2}^{u}=: \theta^{u}$ and so $\cos \theta=\frac{1}{3}\left(1+\cos ^{2} \theta^{y}+\cos ^{2} \theta^{z}\right) \geq \frac{1}{3}$, with equality if and only if $\cos ^{2} \theta^{y}=\cos ^{2} \theta^{z}=0$, that is $T$ is a $J_{u}$-Lagrangian subspace for any $u \perp x$. If $T$ and $T^{\prime}$ have the same quaternionic angle, we use the canonical frames given in (5.8) below for $T$ and for $T^{\prime}$ and define $P$ by $P(B)=B^{\prime}, P\left(B^{\perp}\right)=B^{\prime \perp}$. Then $P J_{u}=J_{u^{\prime}} P$ for $\left(u, u^{\prime}\right)=\left(x, x^{\prime}\right),\left(y, y^{\prime}\right)$ or $\left(z, z^{\prime}\right)$, and $P(X)=X^{\prime}, P(Y)=Y^{\prime}$, and taking $\zeta \in S p(1)$ such that $\tau_{\zeta}$ maps $(x, y, z)$ to $\left(x^{\prime}, y^{\prime}, z^{\prime}\right)$, we get $\xi(\cdot)=P(\cdot) \zeta^{-1} \in S p(V)$, what proves that $P=(\xi, \zeta) \in S p(V) \cdot S p(1)$.

Some further algebraic considerations. Let $T$ be a Euclidean space of dimension 4. For each linear map $l: T \rightarrow T$ we define $\wedge^{2} l: \wedge^{2} T \rightarrow$ $\wedge^{2} T, \wedge^{2} l(u \wedge v)=l(u) \wedge l(v)$. If $\lambda_{i} \geq 0$ are the eigenvalues of $\sqrt{l^{T} l}$ (also called the singular values of $l$ ) and $B=\left\{e_{i}\right\}$ a corresponding orthonormal basis of eigenvectors, let $\tilde{e}_{i}$ defined by $l\left(e_{i}\right)=\lambda_{i} \tilde{e}_{i}$ whenever $\lambda_{i} \neq 0$, and 
extend to an orthonormal basis $\tilde{e}_{i}$ of $T$. Using Newton inequalities we have $2 \sum_{1 \leq i<j \leq 4} \lambda_{i} \lambda_{j} \leq 3\left(\lambda_{1}^{2}+\cdots+\lambda_{4}^{2}\right)$ with equality iff $\lambda_{i}=\lambda_{j} \forall i, j$. Each direct orthonormal basis $B$ of $T$ and $B^{\perp}$ of $T^{\perp}$ define respectively a direct orthonormal basis $\Lambda_{r}^{ \pm}=\Lambda_{r}^{ \pm}(B)$ of $\wedge_{ \pm}^{2} T$, and $\Xi_{r}^{ \pm}$of $\wedge_{ \pm}^{2} T^{\perp}$. We consider the two hyper-Hermitean structures of $T$ (denoted by $J_{s}^{T}$, when we choose one) $J_{r}^{B}=\Lambda_{r}^{+}, \tilde{J}_{r}^{B}=\Lambda_{r}^{-}$. We note the following: If $u, v$ is an o.n. system of vectors of $T$ then

$$
\left|\left\langle u \wedge v, \Lambda_{r}^{+}\right\rangle\right| \leq 1, \quad \text { with equality to } 1 \text { iff } v= \pm J_{r}^{B}(u) .
$$

and similar for $\Lambda_{r}^{-}$. We define

$$
Q^{ \pm} l=-\frac{1}{3} \sum_{r} J_{r}^{T} \circ l \circ J_{r}^{T}, \quad H^{ \pm} l=\frac{1}{4}\left(l+3 Q^{ \pm} l\right)
$$

where \pm depends on $J_{r}^{T}=J_{r}^{B}$ or $\tilde{J}_{r}^{B}$. Note that $H^{ \pm}: S k e w(T) \rightarrow s p_{1}(T)$ gives the orthogonal projection of $l$ onto a $\left(J_{r}^{T}\right)$-hyper-complex linear map (does not depend on the oriented basis $B$ ). We also have $\left\langle l, Q^{ \pm}(l)\right\rangle=$ $\sum_{r=1,2,3} \frac{4}{3}\left\langle\wedge^{2} l\left(\Lambda_{r}^{ \pm}\right), \Lambda_{r}^{ \pm}\right\rangle$and that

$$
\begin{aligned}
& \left|\left\langle\wedge^{2} l\left(\Lambda_{1}^{ \pm}\right), \Lambda_{1}^{ \pm}\right\rangle\right| \leq \frac{1}{2}\left(\lambda_{1} \lambda_{2}+\lambda_{3} \lambda_{4}\right), \\
& \left|\left\langle\wedge^{2} l\left(\Lambda_{2}^{ \pm}\right), \Lambda_{2}^{ \pm}\right\rangle\right| \leq \frac{1}{2}\left(\lambda_{1} \lambda_{3}+\lambda_{2} \lambda_{4}\right), \\
& \left|\left\langle\wedge^{2} l\left(\Lambda_{3}^{ \pm}\right), \Lambda_{3}^{ \pm}\right\rangle\right| \leq \frac{1}{2}\left(\lambda_{1} \lambda_{4}+\lambda_{2} \lambda_{3}\right),
\end{aligned}
$$

and $\|l\|^{2}=\lambda_{1}^{2}+\lambda_{2}^{2}+\lambda_{3}^{2}+\lambda_{4}^{2}$. Moreover,

$$
9\left\|Q^{ \pm} l\right\|^{2}=3\|l\|^{2}-2 \sum_{r}\left\langle J_{r}^{T} \circ l \circ J_{r}^{T}, l\right\rangle=3\|l\|^{2}+6\left\langle Q^{ \pm} l, l\right\rangle,
$$

and so

$$
\begin{aligned}
0 & \leq 16\left\|H^{ \pm} l\right\|^{2}=\left(\|l\|^{2}+6\left\langle Q^{ \pm} l, l\right\rangle+9\left\|Q^{ \pm} l\right\|^{2}\right) \\
& =4\|l\|^{2}+12\left\langle Q^{ \pm} l, l\right\rangle=16\left\langle H^{ \pm} l, l\right\rangle .
\end{aligned}
$$

Consequently $3\left\langle Q^{ \pm} l, l\right\rangle \geq-\|l\|^{2}$. If equality holds, then $H^{ \pm} l=0$, and so $l \in \operatorname{sp}_{1}(T)^{\perp}=\wedge_{+}^{2} T=\operatorname{span}\left\{J_{r}^{T}\right\}$. Newton inequalities and (5.6) prove that

$$
\begin{gathered}
-\frac{1}{3}\|l\|^{2} \leq\left\langle Q^{ \pm} l, l\right\rangle \leq\|l\|^{2}, \\
\left\langle Q^{ \pm} l, l\right\rangle=\|l\|^{2} \text { iff } l \text { is hyper-complex, } \\
\left\langle Q^{ \pm} l, l\right\rangle=-\frac{1}{3}\|l\|^{2} \text { iff } l \in \wedge_{+}^{2} T=\operatorname{span}\left\{J_{s}^{T}\right\}
\end{gathered}
$$

Furthermore, if $l$ is hyper-complex then $l$ is conformal. The singular values of $\wedge^{2} l$ are $\lambda_{i} \lambda_{j}$ for $i<j$. We can split

$$
\wedge^{2} l=\wedge_{+}^{+} l \oplus \wedge_{-}^{+} l \oplus \wedge_{+}^{-} l \oplus \wedge_{-}^{-} l,
$$

where $\wedge_{+}^{ \pm} l: \wedge_{+}^{2} T \rightarrow \wedge_{ \pm}^{2} T$ and $\wedge_{-}^{ \pm} l: \wedge_{-}^{2} T \rightarrow \wedge_{ \pm}^{2} T$. 
$\wedge^{2} l$ is self dual (resp. anti-self-dual), i.e. $\wedge^{2} l *=* \wedge^{2} l\left(\right.$ resp. $\left.\wedge^{2} l *=-* \wedge^{2} l\right)$ iff the anti-self dual part $\wedge_{+}^{-} l \oplus \wedge_{-}^{+} l$ vanish (resp. the self-dual part $\wedge_{+}^{+} l \oplus \wedge_{-}^{-} l$ vanish), iff either $\wedge^{2} l=0$, what means at least 3 of the singular values vanish, or $l$ is an orientation preserving (resp. reversing) conformal isomorphism.

If $(\bar{M}, g, Q)$ is a quaternionic-Kähler manifold of real dimension $4 n$ and fundamental form $\Omega$, the quaternionic $4 m$-submanifolds, are necessarily totally geodesic $([16])$. Some attention have been drawn to a more general type of submanifolds, the almost complex submanifolds in the quaternionic context, and their minimality have been studied. This includes the quaternionic submanifolds as well the totally complex or the Kähler submanifolds. See for example [1] and their references, where some examples can be found. Most of these submanifolds are also proved to be totally geodesic. We will show some use of the quaternionic angle in the study of almost complex submanifolds with parallel mean curvature.

An immersed submanifold $F: M \rightarrow \bar{M}$ is an almost complex submanifold if there exist a smooth section $J_{M}: M \rightarrow Q$ such that, for each $p \in M$, $J_{M}(p)\left(T_{p} M\right) \subset T_{p} M$. If $n=2$ and $m=1$, the quaternionic angle satisfies $\frac{1}{3} \leq \cos \theta \leq 1$ with equality to $\frac{1}{3}$ at totally complex points and to 1 at quaternionic points. Since $\cos \theta \geq \frac{1}{3}$ we conclude from Theorem 1.4:

Proposition 5.6. If $(\bar{M}, g, Q)$ is a quaternionic-Kähler manifold of real dimension 8 and $M$ is an almost complex complete submanifold of real dimension 4 and with parallel mean curvature and Ricci $^{M} \geq 0$, then $M$ is a minimal submanifold.

For an almost complex 4-dimensional submanifold, $\Phi: T M \rightarrow N M$ is a conformal morphism with coefficient of conformality $(1-\cos \theta)\left(\cos \theta-\frac{1}{3}\right)$ ([33]). To see this we first note that we can take canonical orthonormal basis $B$ of $T_{p} M$ and $B^{\perp}$ of $N M_{p}$ of the form

$$
\begin{aligned}
B & =\left\{X, J_{x} X, c J_{y} X+s Y, c J_{z} X+s J_{x} Y\right\}=\left\{X_{k}\right\} \\
B^{\perp} & =\left\{J_{y} Y, J_{z} Y, c Y-s J_{y} X, c J_{x} Y-s J_{z} X\right\}=\left\{U_{i}\right\}
\end{aligned}
$$

where $c^{2}+s^{2}=1, x, y, z=x \times y$ is an o.n. basis of $\mathbb{R}^{3}$ with $J_{x}=J_{M}(p)$ and $Y \in H_{X}^{\perp}$. Then

$$
\cos \theta=\left(1-\frac{2}{3} s^{2}\right), \quad s^{2}=\frac{3}{2}(1-\cos \theta), \quad c^{2}=\frac{3}{2}\left(\cos \theta-\frac{1}{3}\right),
$$

and using this basis we see that $\Phi(B)=-\frac{2}{3} s c B^{\perp}$.

Next we use the formula of $\Delta \cos \theta$ to obtain some nonexistence results for almost complex submanifolds, and in particular to give a "calibration"type proof of the above mentioned result of Gray [16], for the case $n=2$ and 
$m=1$. We take for basis of $N M_{p}$, that is reordering $B^{\perp}, B^{\prime \perp}=\left\{U_{i}^{\prime}\right\}, U_{1}^{\prime}=$ $U_{3}, U_{2}^{\prime}=U_{4}, U_{3}^{\prime}=U_{1}, U_{4}^{\prime}=U_{2}$, and consider the corresponding basis $\Xi_{t}^{\prime \pm}$, of $\wedge^{2} N M_{p}$. The matrix of $\Psi: \wedge^{2} T M \rightarrow \wedge^{2} N M$, with respect to the basis $\Lambda_{1}^{+}, \Lambda_{2}^{+}, \Lambda_{3}^{+}, \Lambda_{1}^{-}, \Lambda_{2}^{-}, \Lambda_{3}^{-}$of $\wedge^{2} T M$ and the basis $\Xi_{1}^{\prime+}, \Xi_{2}^{\prime+}, \Xi_{3}^{\prime+}, \Xi_{1}^{\prime-}, \Xi_{2}^{\prime-}, \Xi_{3}^{\prime-}$ of $\wedge^{2} N M_{p}$ is given by

$$
\Psi=\left[\begin{array}{cccccc}
\frac{2}{3}\left(1+s^{2}\right) & 0 & 0 & 0 & 0 & 0 \\
0 & \frac{2}{3} c^{2} & 0 & 0 & 0 & 0 \\
0 & 0 & \frac{2}{3} c^{2} & 0 & 0 & 0 \\
0 & 0 & 0 & \frac{2}{3} s^{2} & 0 & 0 \\
0 & 0 & 0 & 0 & \frac{2}{3} s^{2} & 0 \\
0 & 0 & 0 & 0 & 0 & -\frac{2}{3} s^{2}
\end{array}\right]
$$

Note that $w_{M}=\sqrt{2} \Lambda_{1}^{+}$and $w_{N M}=\sqrt{2} \Xi_{1}^{\prime+}$ are the respective Kähler forms. $\Psi$ applies $\wedge_{ \pm}^{2} T M$ into $\wedge_{ \pm}^{2} N M$ and denoting the corresponding restriction $\Psi_{ \pm}: \wedge_{ \pm}^{2} T M \rightarrow \wedge_{ \pm}^{2}(N M)$, and defining $\Psi_{+}^{\prime}:=\Psi_{+}-2(1-\cos \theta) \Psi_{0}$, where $\Psi_{0}: \wedge^{2} T M \rightarrow \wedge^{2} N M$ is the linear morphism given by $\Psi_{0}\left(w_{M}\right)=w_{N M}$ and zero on the orthogonal complement of $\mathbb{R} w_{M}$, then $\Psi_{+}^{\prime}$ and $\Psi_{-}$are conformal, with $\left\|\Psi_{+}^{\prime}(\eta)\right\|^{2}=\left(\cos \theta-\frac{1}{3}\right)^{2}\|\eta\|^{2}, \forall \eta \in \wedge_{+}^{2} T M$, and $\left\|\Psi_{-}(\eta)\right\|^{2}=(1-$ $\cos \theta)^{2}\|\eta\|^{2}, \forall \eta \in \wedge_{-}^{2} T M$. Thus, if $M$ is immersed with no totally complex points the bundles $\wedge_{+}^{2} T M$ and $\wedge_{+}^{2} M N$ are isomorphic. If $M$ is immersed with no quaternionic points, then $\wedge_{-}^{2} T M$ and $\wedge_{-}^{2} M N$ are isomorphic. If there are neither quaternionic nor totally complex points, then $\Phi: T M \rightarrow$ $N M$ is an isomorphism.

If $X_{i}$ is a direct o.n. basis of $T_{p} M$ and $Y_{i} \in N M_{p}$ are any vectors, then

$\sum_{s<j} \Omega\left(X_{1}, \ldots, Y_{s(s)}, \ldots, Y_{j_{(j)}}, \ldots, X_{4}\right)=\sum_{r} \Omega\left(\Lambda_{r}^{+}, \Lambda_{r}^{+}(Y)\right)-\Omega\left(\Lambda_{r}^{-}, \Lambda_{r}^{-}(Y)\right)$

with $* \Lambda_{r}^{-}=-\Lambda_{r}^{-}$, and where $\Lambda_{r}^{ \pm}(Y), Y=\left(Y_{1}, \ldots, Y_{4}\right)$, are formally defined in the same way as $\Lambda_{r}^{ \pm}(B)$. Thus, we consider the two components of $\wedge^{2} B$, $\wedge_{+}^{+} B: \wedge_{+}^{2} T_{p} M \rightarrow \wedge_{+}^{2} N M_{p}$ and $\wedge_{-}^{-} B: \wedge_{-}^{2} T_{p} M \rightarrow \wedge_{-}^{2} N M_{p}$, and say that $\wedge^{2} B$ is self-dual iff $\wedge^{2} B=\wedge_{+}^{+} B \oplus \wedge_{-}^{-} B$ and it is anti-self-dual if the self dual part vanish. Therefore, from lemma 3.1

$$
\begin{aligned}
\Delta \cos \theta= & -\cos \theta\|B\|^{2}+2\left\langle\Psi_{+}, \wedge_{+}^{+} B\right\rangle+2\left\langle\Psi_{-}, \wedge_{-}^{-} B\right\rangle \\
& +m\left\langle\nabla^{\perp} H, \Phi\right\rangle-\sum_{i j} \bar{R}\left(X_{i}, X_{k}, X_{i}, \Phi\left(X_{k}\right)\right)
\end{aligned}
$$

Now we prove the classic result on quaternionic submanifolds in [16] reducing it to a linear algebra problem:

Proposition 5.7. If $M$ is a quaternionic submanifold of $\bar{M}$ then $M$ is totally geodesic. 
Proof. We identify $T_{p} M=H_{X} \equiv H_{Y}=N M_{p}$, through the canonical basis $B=\left\{X, J_{x} X, J_{y} X, J_{z} X\right\}=\left\{X_{k}\right\}$ and $\left(B^{\prime}\right)^{\perp}=\left\{Y, J_{x} Y, J_{y} Y, J_{z} Y\right\}$, and set $l_{k}=B\left(X_{k}, \cdot\right): T_{p} M \rightarrow N M_{p} \equiv T_{p} M$. We have $\Phi=0$ and $\Psi_{-}=0$, $\Psi_{+}=\frac{2}{3} I d$. Then (5.10) is $0=\sum_{k}\left\langle Q_{B}^{ \pm}\left(l_{k}\right), l_{k}\right\rangle-\left\|l_{k}\right\|^{2}$. By (5.7) each $l_{k}$ is hyper-complex, that is $B\left(X_{k}, J_{x} Z\right)=J_{x}\left(B\left(X_{k}, Z\right)\right), \forall x \in \mathbb{S}^{2}$. Consequently, $B\left(J_{x} Z, J_{x} W\right)=J_{x}^{2} B(Z, W)=-B(Z, W)$, for any $x$. But then $B(Z, W)=-B\left(J_{y} Z, J_{y} W\right)=B\left(J_{x} J_{y} Z, J_{x} J_{y} W\right)=B\left(J_{z} Z, J_{z} W\right)$, for any o.n. basis $x, y, z=x \times y$ of $\mathbb{R}^{3}$ and so $B=0$.

At $p$ consider the canonical frames $B=\left\{X_{k}\right\}$ and $B^{\prime \perp}=\left\{U_{k}^{\prime}\right\}$, and the linear isometry $L: N M_{p} \rightarrow T_{p} M, L\left(U_{k}^{\prime}\right)=X_{k}$. We define $l_{k}=L \circ B\left(X_{k}, \cdot\right)$ : $T_{p} M \rightarrow T_{p} M$, and $l_{k}^{\prime}=l_{k} \circ S^{\prime}$ and $l_{k}^{\prime \prime}=l_{k} \circ S^{\prime \prime}$, where $S^{\prime}, S^{\prime \prime}$ are orientation preserving isometries, by

$$
\begin{gathered}
l_{k}^{\prime}\left(X_{1}\right)=l_{k}\left(X_{1}\right), l_{k}^{\prime}\left(X_{2}\right)=l_{k}\left(X_{2}\right), l_{k}^{\prime}\left(X_{3}\right)=-l_{k}\left(X_{3}\right), l_{k}^{\prime}\left(X_{4}\right)=-l_{k}\left(X_{4}\right) \\
l^{\prime \prime}{ }_{k}\left(X_{1}\right)=l_{k}\left(X_{1}\right), l^{\prime \prime}{ }_{k}\left(X_{2}\right)=-l_{k}\left(X_{2}\right), l_{k}^{\prime \prime}{ }_{k}\left(X_{3}\right)=-l_{k}\left(X_{3}\right), l^{\prime \prime}{ }_{k}\left(X_{4}\right)=l_{k}\left(X_{4}\right) .
\end{gathered}
$$

Then, $\left\|l_{k}^{\prime}\right\|=\left\|l^{\prime \prime}{ }_{k}\right\|=\left\|l_{k}\right\|=\left\|B\left(X_{k}, \cdot\right)\right\|$, and we have

$$
\begin{aligned}
& \wedge^{2} l_{k}^{\prime}\left(\Lambda_{1}^{ \pm}\right)=\wedge^{2} l_{k}\left(\Lambda_{1}^{ \pm}\right), \wedge^{2} l_{k}^{\prime}\left(\Lambda_{2}^{ \pm}\right)=-\wedge^{2} l_{k}\left(\Lambda_{2}^{ \pm}\right), \wedge^{2} l_{k}^{\prime}\left(\Lambda_{3}^{ \pm}\right)=-\wedge^{2} l_{k}\left(\Lambda_{3}^{ \pm}\right), \\
& \wedge^{2} l^{\prime \prime}{ }_{k}\left(\Lambda_{1}^{ \pm}\right)=-\wedge^{2} l_{k}\left(\Lambda_{1}^{ \pm}\right), \wedge^{2} l^{\prime \prime}{ }_{k}\left(\Lambda_{2}^{ \pm}\right)=-\wedge^{2} l_{k}\left(\Lambda_{2}^{ \pm}\right), \wedge^{2} l^{\prime \prime}{ }_{k}\left(\Lambda_{3}^{ \pm}\right)=\wedge^{2} l_{k}\left(\Lambda_{3}^{ \pm}\right) .
\end{aligned}
$$

Set

$$
\begin{aligned}
D & =\sum_{k}\left(\left\|l_{k}\right\|^{2}-\left\langle Q^{+} l_{k}, l_{k}\right\rangle\right) \geq 0 \\
A & =\sum_{k}\left(\left\langle Q^{+} l_{k}^{\prime}, l_{k}^{\prime}\right\rangle+\frac{1}{3}\left\|l_{k}\right\|^{2}\right) \geq 0 \\
E & =\sum_{k}\left(\left\langle Q^{-} l^{\prime \prime}{ }_{k}, l^{\prime \prime}{ }_{k}\right\rangle+\frac{1}{3}\left\|l_{k}\right\|^{2}\right) \geq 0
\end{aligned}
$$

Note that $\wedge^{2} B=\sum_{k} \wedge^{2} l_{k}$ and is antiselfdual iff $\sum_{k} \wedge_{-}^{+} l_{k} \oplus \wedge_{+}^{-} l_{k}=0$. By (5.7)

Lemma 5.4. At $p, 0 \leq D, A, E \leq \frac{4}{3}\|B\|^{2}$. Furthermore, $D=0$ iff $A($ or $E)=\frac{4}{3}\|B\|^{2}$ iff $B=0$. If $A=0(E=0$ resp. $)$ then $\wedge^{2} B$ is selfdual (resp. antiselfdual).

Now we investigate when $\tilde{Q}_{\Omega}(B) \geq \delta\|B\|^{2}$. Using the matrix (5.9)

$$
\begin{aligned}
\cos \theta & \tilde{Q}_{\Omega}(B)=\cos \theta\|B\|^{2}-2\left\langle\Psi_{+}, \wedge_{+}^{+} B\right\rangle-2\left\langle\Psi_{-}, \wedge_{-}^{-} B\right\rangle \\
= & -\sum_{k}\left(\sum_{r=1,2,3} \frac{4}{3}\left\langle\Lambda_{r}^{+}, \wedge^{2} l_{k}\left(\Lambda_{r}^{+}\right)\right\rangle-\left\|l_{k}\right\|^{2}\right) \\
& -s^{2} \sum_{k}\left(\frac{4}{3}\left\langle\Lambda_{1}^{+}, \wedge^{2} l_{k}\left(\Lambda_{1}^{+}\right)\right\rangle-\frac{4}{3}\left\langle\Lambda_{2}^{+}, \wedge^{2} l_{k}\left(\Lambda_{2}^{+}\right)\right\rangle-\frac{4}{3}\left\langle\Lambda_{3}^{+}, \wedge^{2} l_{k}\left(\Lambda_{3}^{+}\right)\right\rangle\right. \\
& \left.+\frac{4}{3}\left\langle\Lambda_{1}^{-}, \wedge^{2} l_{k}\left(\Lambda_{1}^{-}\right)\right\rangle+\frac{4}{3}\left\langle\Lambda_{2}^{-}, \wedge^{2} l_{k}\left(\Lambda_{2}^{-}\right)\right\rangle-\frac{4}{3}\left\langle\Lambda_{3}^{-}, \wedge^{2} l_{k}\left(\Lambda_{3}^{-}\right)\right\rangle+\frac{2}{3}\left\|l_{k}\right\|^{2}\right) \\
= & \left(D+s^{2} E\right)-s^{2}\left(A+\frac{2}{3}\|B\|^{2}\right) .
\end{aligned}
$$


Lemma 5.5. Assume at $p$, for each $k,\left\|H^{+} l_{k}\right\| \leq \epsilon\left\|l_{k}\right\|$ where $0 \leq \epsilon \leq 1$, and $0 \leq \tau \leq \frac{4(1-\epsilon)}{9}$ such that $(1-\cos \theta) \leq \tau$. Then, at $p$,

$$
\cos \theta \tilde{Q}_{\Omega}(B) \geq \delta\|B\|^{2}
$$

where $\delta=\frac{4(1-\epsilon)-9 \tau}{3} \geq 0$.

Note that we assume $\cos \theta \geq \frac{5}{9}$. Lemma 5.5 includes the case $\cos \theta \equiv 1$, that implies $l_{k}$ hypercomplex, giving $\epsilon=1$ and $\delta=0$, as in proof of Proposition 5.7.

Proof. The condition on $H^{+} l_{k}$ implies

$$
\left.\left.\left|\left\langle Q^{+} l_{k}, l_{k}\right\rangle+\frac{1}{3}\right| l_{k}\right|^{2}\left|\leq \frac{4}{3} \epsilon\right| l_{k}\right|^{2} .
$$

Then

$$
D=\sum_{k} \frac{4}{3}\left\|l_{k}\right\|^{2}-\left(\frac{1}{3}\left\|l_{k}\right\|^{2}+\left\langle Q^{+} l_{k}, l_{k}\right\rangle\right) \geq \frac{4}{3}(1-\epsilon)\|B\|^{2} .
$$

Using the bounds in lemma 5.4 and (5.15), we obtain

$$
\cos \theta \tilde{Q}_{\Omega}(B) \geq \frac{4(1-\epsilon)-6 s^{2}}{3}\|B\|^{2}
$$

that proves the lemma.

Note that $(1.7)$ can be satisfied. If $\bar{M}$ is a quaternionic space form of reduced scalar curvature $\nu=s^{\bar{M}} / 32$, then

$\bar{R}(X, Y, Z, W)=\frac{\nu}{4}\left(\langle X \wedge Y, Z \wedge W\rangle+\sum_{r}\left\langle J_{r} X \wedge J_{r} Y, Z \wedge W\right\rangle+\left\langle J_{r} X, Y\right\rangle\left\langle J_{r} Z, W\right\rangle\right)$

and so

$$
\sum_{k i} \bar{R}\left(X_{i}, X_{k}, X_{i}, \Phi\left(X_{k}\right)\right)=9 \nu(\cos \theta)\left(\cos \theta-\frac{1}{3}\right)=4 \nu s^{2} c^{2}
$$

Proposition 5.8. Assume $F: M \rightarrow \bar{M}$ is a closed almost complex immersed submanifold such that (1.7) holds.

(1) If there exist constants $0 \leq \epsilon \leq 1,0 \leq \tau<4(1-\epsilon) / 9$, such that $(1-\cos \theta) \leq \tau$ and at each point $p \in M$ there exist canonical frames $B$ and $B^{\perp}$ such that $\left\|H^{+} l_{k}\right\| \leq \epsilon\left\|l_{k}\right\|$, then $F$ is totally geodesic.

Furthermore, if $\bar{M}$ is a quaternionic space form then:

(2) If $F: M \rightarrow \bar{M}$ is parallel and $\nu \neq 0$ then either $F$ is a quaternionic submanifold or a totally complex submanifold.

(3) If $M$ is closed, $F$ has parallel mean curvature and $\wedge^{2} B$ is anti-self-dual then $F$ is totally geodesic and if $\nu>0$ then either $F$ is totally complex or a quaternionic submanifold. 
Proof. (1) follows from previous lemma and Theorem 1.5(A). If we assume $\tau<\frac{4(1-\epsilon)}{9}$, it guarantees $\delta>0$. (2) From the proof of Proposition 3.2,

$$
\sum_{k}\left(\bar{R}\left(X_{k}, X_{i}\right) X_{k}\right)^{\perp}=0
$$

and by (5.16),

$$
4 \nu s^{2} c^{2}=9 \nu(1-\cos \theta)\left(\cos \theta-\frac{1}{3}\right)=0
$$

(3) We have

$$
\Delta \cos \theta=-4 \nu s^{2} c^{2}-\cos \theta\|B\|^{2} \leq 0,
$$

what implies $\cos \theta \geq \frac{1}{3}$ is constant and so $-4 \nu s^{2} c^{2}-\cos \theta\|B\|^{2}=0$.

Proposition 5.9. Assume $M$ is a closed almost complex submanifold with parallel mean curvature on a quaternionic space form $\bar{M}$. Then $F$ is totally geodesic if (1) or (2) below holds:

(1) $\nu>0$ and $\|B\|^{2} \leq 3 \nu\left(\cos \theta-\frac{1}{3}\right)$

(2) $\nu<0$ and $\|B\|^{2} \leq-\frac{27}{8} \nu\left(\cos \theta-\frac{1}{3}\right)(1-\cos \theta)$.

Proof. We may write $\Delta \cos \theta$ given in (5.10) and using (5.15) as

$$
\Delta s^{2}=6 \nu s^{2} c^{2}+4 s c \sum_{j}\left\langle\nabla_{X_{j}}^{\perp} H, U_{j}\right\rangle-\frac{3}{2} s^{2}\left(A+\frac{2}{3}\|B\|^{2}\right)+\frac{3}{2}\left(D+s^{2} E\right) .
$$

(1) The conditions imply

$$
\frac{3}{2} s^{2}\left(A+\frac{2}{3}\|B\|^{2}\right) \leq 6 \nu s^{2} c^{2}
$$

and so $\Delta s^{2} \geq 0$.

(2) Under the assumptions,

$$
\frac{3}{2}\left(D+s^{2} E\right) \leq 2\left(1+s^{2}\right)\|B\|^{2} \leq 4\|B\|^{2} \leq-6 s^{2} c^{2} \nu
$$

and we have $\Delta s^{2} \leq 0$. In both cases (1)(2) we conclude that $s$ is constant, and again that $\Delta s^{2}=0$. This implies in case (1)

$$
D+s^{2} E=0
$$

and in case (2)

$$
s^{2}\left(A+\frac{2}{3}\|B\|^{2}\right)=0
$$

what leads to the conclusion in the proposition (see Lemma 5.4). 


\subsection{The special calibrations}

The special Lagrangian calibration. Let $(\bar{M}, g, J, \rho)$ be a Calabi-Yau manifold of complex dimension $k$ with holomorphic volume element $\rho \in \wedge^{(k, 0)} M$. Then $\operatorname{Re}(\rho)$ is the Lagrangian calibration and calibrates the special Lagrangian submanifolds. On $\bar{M}$ it is also defined the Kähler calibrations. If $k=4$, there is also a $S^{1}$-family of Cayley calibrations

$$
\Omega_{\theta}=-\frac{1}{2} w^{2}+R e\left(e^{\theta} \rho\right)
$$

that calibrates the Cayley 4-submanifolds.

The Cayley calibration. If $\left(\bar{M}^{8}, \bar{g}, \Omega\right)$ is a $\operatorname{Spin}(7)$ 8-dimensional manifold, then it is defined a Cayley calibration $\Omega$. Given a $\operatorname{Spin}(7)$-frame $e_{i}$ that identifies $T_{p} \bar{M}$ with the space of octonions $\mathbb{R}^{8}, \Omega$ is the 4 -form defined by $\Omega(x, y, z, w)=\langle x, y \times z \times w\rangle$, where the cross product of three vectors is defined in $\mathbb{R}^{8}$. A Calabi-Yau 4-fold is also a $\operatorname{Spin}(7)$ manifold and any Cayley calibrations defined above corresponds to this definition. If $F: M^{4} \rightarrow \bar{M}$ is an immersed 4-submanifold, then $\Phi\left(X_{1}\right)=\left(X_{2} \times X_{3} \times X_{4}\right)^{\perp}$, where $X_{i}$ is a d.o.n. basis of $T_{p} M$.

The associative and the co-associative calibration. Let $\left(\bar{M}^{7}, \bar{g}, \phi\right)$ be a $G_{2}$ Riemannian manifold with a closed $G_{2}$ 3-form $\phi$. Identifying $T_{p} \bar{M}$ with $\mathbb{R}^{7}=\operatorname{Im}\left(\mathbb{R}^{8}\right)$ by a $G_{2}$-frame, $\phi(x, y, z)=\langle x, y z\rangle$ where on the right hand side it is considered the octonion product. This is the associative calibration. The co-associative calibration is $\psi=* \phi$ and satisfies $\psi(x, y, z, w)=\frac{1}{2}\langle x,[y, z, w]\rangle$ where $[y, z, w]=(y z) w-y(z w)$ is the associator operator. The forms $\phi$ and $\psi$ calibrate respectively the associative 3-dimensional submanifolds and the co-associative 4-dimensional submanifolds. If $F: M \rightarrow \bar{M}$ is an immersed 3-submanifold, $\Phi_{\phi}\left(X_{1}\right)=\left(X_{2} X_{3}\right)^{\perp}$, where $X_{1}, X_{2}, X_{3}$ is any d.o.n. basis of $T_{p} M$. If $F$ is an immersed 4-submanifold, $\Phi_{\psi}\left(X_{1}\right)=\left[X_{2}, X_{3}, X_{4}\right]^{\perp}$. If $N$ is a Calabi-Yau 3-fold, then $N \times S^{1}$ or $N \times \mathbb{R}$ are $G_{2}$-manifolds with

$$
\phi=1^{*} \wedge w+\operatorname{Re}(\rho) \quad \text { and } \quad \psi=\frac{1}{2} w \wedge w-1^{*} \wedge \operatorname{Im}(\rho) .
$$

If $N$ is a $G_{2}$ manifold, then $N \times S^{1}$ or $N \times \mathbb{R}$ with $\Omega=1^{*} \wedge \phi+\psi$ are $\operatorname{Spin}(7)$ manifolds.

Acknowledgements. The authors would like to thank the referee for helpful comments and valuable suggestions. The second author would like to thank Dmitri Alekseevsky for many conversations in quaternionic Kähler geometry. 


\section{References}

[1] Alekseevsky, D. V. and Marchiafava, S.: Hermitean and Kähler submanifolds of a quaternionic Kähler manifold. Osaka J. Math. 38 (2001), no. 4, 869-904.

[2] Almgren, F. J., JR.: Some interior regularity theorems for minimal surfaces and an extension of Bernstein's theorem. Ann. of Math. (2) 84 (1966), 277-292.

[3] Alías, L., Dajczer, M. and Ripoll, J.: A Bernstein-type theorem for Riemannian manifolds with a Killing field. Ann. Global Anal. Geom. 31 (2007), no. 4, 363-373.

[4] Barbosa, J. L., Bessa, G.P. And Montenegro, J.F.: BernsteinHeinz-Chern-Flanders inequalities. Math. Proc. Cambridge Philos. Soc. 144 (2008), no. 2, 457-464.

[5] Barbosa, J. L., Kenmotsu, K. and Oshikiri, G.: Foliations by hypersurfaces with constant mean curvature. Math. Z. 2007 (1991), no. 1, 97-107.

[6] Bernstein, S. N.: Über ein geometrisches Theorem und seine Anwendung auf die partiellen Differentialgleichungen vom elliptischen Typus. Math. Z. 26 (1927), no. 1, 551-558.

[7] Chavel, I.: Eigenvalues in Riemannian geometry. Pure and Applied Mathematics 115. Academic Press, Orlando, FL, 1984.

[8] Cheng, S. Y.: Eigenvalue comparison theorems and its geometric applications. Math. Z. 143 (1975), no. 3, 289-297.

[9] Chern, S. S.: On the curvatures of a piece of hypersurface in euclidean space. Abh. Math. Sem. Univ. Hamburg 29 (1965), 77-91.

[10] Chern, S. S.: Simple proofs of two theorems on minimal surfaces. Enseignement Math. (2) 15 (1969), 53-61.

[11] De Giongi, E.: Una estensione del teorema di Bernstein. Ann. Scuola Norm. Sup. Pisa (3) 19 (1965), 79-85.

[12] Do Carmo, M. and Peng, C. K.: Stable complete minimal surfaces in $\mathbb{R}^{3}$ are planes. Bull. Amer. Math. Soc. (N.S.) 1 (1979), no. 6, 903-906.

[13] Ecker, K. And Huisken, G.: A Bernstein result for minimal graphs of controlled growth. J. Differential Geom. 31 (1990), no. 2, 397-400.

[14] Fischer-Colbrie, D. And Schoen, R.: The structure of complete stable minimal surfaces in 3-manifolds of nonnegative scalar curvature. Comm. Pure Appl. Math. 33 (1980), no. 2, 199-211.

[15] Flanders, H.: Remark on mean curvature. J. London Math. Soc. 41 (1966), 364-366.

[16] Gray, A.: A note on manifolds whose holonomy group is a subgroup of $S p(n) \cdot S p(1)$. Michigan Math. J. 16 (1969), 125-128. 
[17] Harvey, R. and Lawson, H. B. JR.: Calibrated geometries. Acta Math. 148 (1982), 47-157.

[18] Heinz, E.: Über Flächen mit eineindeutiger Projektion auf eine Ebene, deren Krümmungen durch Ungleichungen eingeschränkt sind. Math. Ann. 129 (1955), 451-454.

[19] Hildebrandt, S., Jost, J. and Widman, K. O.: Harmonic mappings and minimal submanifolds. Invent. Math. 62 (1980/81), no. 2, 269-298.

[20] Hirakawa, S.: Constant Gaussian curvature surfaces with parallel mean curvature vector in two-dimensional complex space forms. Geom. Dedicata 118 (2006), 229-244.

[21] Hoffman, D. And Spruck, J.: Sobolev and isoperimetric inequalities for Riemannian submanifolds. Comm. Pure Appl. Math. 27 (1974), 715-727.

[22] Joyce, D.: Compact manifolds with special holonomy. Oxford Mathematical Monographs. Oxford University Press, Oxford, 2000.

[23] Kenmotsu, K. and Zhou, D.: The classification of the surfaces with parallel mean curvature vector in two-dimensional complex space forms. Amer. J. Math. 122 (2000), no. 2, 295-317.

[24] Kraines, Y.: Topology of quaternionic manifolds. Trans. Amer. Math. Soc. 122 (1966), 357-367.

[25] Li, G. and Salavessa, I.: Graphic Bernstein results in curved pseudoRiemannian manifolds. J. Geom. Phys. 59 (2009), no. 9, 1306-1313.

[26] LI, P.: Lecture notes on geometric analysis. Lecture Notes Series 6. Seoul National University, Research Institute of Mathematics, Global Analysis Research Center, Seoul, 1993,

[27] Miranda, M.: Una maggiorazione integrale per le curvature delle ipersuperfici minimali. Rend. Sem. Mat. Univ. Padova 38 (1967), 91-107.

[28] Rigoli, M. and Salavessa, I.: Conformal and isometric immersions of Riemannian manifolds. Math. Z. 196 (1987), no. 2, 293-300.

[29] Salavessa, I.: Graphs with parallel mean curvature and a variational problem in conformal geometry. Ph.D. Thesis, University of Warwick, 1987.

[30] Salavessa, I.: Graphs with parallel mean curvature. Proc. Amer. Math. Soc. 107 (1989), no. 2, 449-458.

[31] Salavessa, I.: On the Kähler angles of submanifolds. Port. Math. (N.S.) 60 (2003), no. 2, 215-235.

[32] Salavessa, I.: Spacelike graphs with parallel mean curvature. Bull. Belg. Math. Soc. Simon Stevin 15 (2008), no. 1, 65-76.

[33] Salavessa, I.: Residue formulas for minimal submanifolds. Publ. Real. Soc. Mat. Esp. (XVIWGP2006, Tenerife, Spain) 11 (2007), 353-358.

[34] Salavessa, I. and Valli, G.: Minimal submanifolds of Kähler-Einstein manifolds with equal Kähler angles. Pacific J. Math. 205 (2002), no. 1, 197-235. 
[35] Schoen, R., Simon, L. and Yau, S. T.: Curvature estimates for minimal hypersurfaces. Acta Math. 134 (1975), no. 3-4, 275-288.

[36] Simons, J.: Minimal varieties in riemannian manifolds. Ann. of Math. (2) 88 (1968), 62-105.

[37] Wang, M.: On graphic Bernstein type results in higher codimension. Trans. Amer. Math. Soc. 355 (2003), no. 1, 265-271.

[38] YAU, S. T.: Some function-theoretic properties of complete Riemannian manifolds and their applications to geometry. Indiana Univ. Math. J. 25 (1976), no. 7, 659-670.

[39] YAU, S. T.: Isoperimetric constants and the first eigenvalue of a compact Riemannian manifold. Ann. Sci. École Norm. Sup. (4) 8 (1975), no. 4, 487-507.

Recibido: 3 de octubre de 2008

Revisado: 10 de febrero de 2009

Guanghan Li

School of Mathematics and Computer Science

Hubei University

Wuhan 430062, P. R. China

liguanghan@163.com

Isabel M. C. Salavessa

Centro de Física das Interacções Fundamentais Instituto Superior Técnico, Technical University of Lisbon Edifício Ciência, Piso 3 Av. Rovisco Pais, 1049-001 Lisboa, Portugal isabel.salavessa@ist.utl.pt

The first author is partially supported by NSFC (No. 10501011) and by Fundação Ciência e Tecnologia (FCT) through a FCT fellowship SFRH/BPD/26554/2006. The second author is partially supported by FCT through the Plurianual of CFIF and POCI$\mathrm{PPCDT} / \mathrm{MAT} / 60671 / 2004$. 